\title{
SEMIGROUPS OF UNBOUNDED LINEAR OPERATORS IN BANACH SPACE
}

\author{
BY \\ RHONDA JO HUGHES(l)
}

\begin{abstract}
One-parameter families of unbounded linear operators acting in a Banach space $X$, and satisfying the semigroup and strong continuity properties on a suitable subspace of $X$, are discussed; the notion of infinitesimal generator is generalized to this unbounded setting, and a HilleYosida-type theorem is proved. The theory is illustrated by several examples, which include fractional integrals and derivatives acting in $L^{P}(0, \infty)$.
\end{abstract}

1. Introduction. A central feature of the classical theory of semigroups of bounded linear operators on Banach space is the concept of the infinitesimal generator. The determination of the semigroup in terms of its generator, and the characterization of those operators which act as generators of semigroups, are crucial problems; the Hille-Yosida theorem provides a solution to the latter, and plays a fundamental role in the theory of linear partial differential equations.

Devinatz [5] and Nussbaum [20] and [21] extended the notion of semigroup to include certain one-parameter families of unbounded (symmetric or selfadjoint) operators acting in Hilbert space, which satisfy the semigroup property and are weakly continuous on a suitable linear manifold; a classical example is provided by the Riesz potential operators in $L^{2}\left(R^{n}\right)$ (cf. [21]). Their results yield integral representations of the given semigroups and thus suggest the possibility of expressing the semigroup in terms of an "infinitesimal generator." Since interesting examples of one-parameter families of unbounded linear operators which satisfy the semigroup property are encountered in Banach space (e.g., the classical Riemann-Liouville fractional integral in $L^{p}(0, \infty), 1<p<\infty$; cf. Theorem 3.3), it is natural to ask whether a unified approach to semigroups of unbounded operators is feasible without the aid of spectral representations and the structure of Hilbert space. Thus, in an

Received by the editors June 3, 1975 and, in revised form, October 15, 1975.

AMS (MOS) subject classifications (1970). Primary 47D05; Secondary 26A33.

Key words and phrases. Semigroup of bounded operators, closed operator, resolvent, infinitesimal generator, fractional integral, fractional derivative, Riemann-Liouville semigroup, fractional powers of closed operators.

(1) This paper is based on part of the author's Ph.D. dissertation written under the direction of Professor Shmuel Kantorovitz and submitted to the University of Illinois at Chicago Circle. 
"unbounded" setting, we consider the fundamental problems of classical semigroup theory mentioned earlier.

In $\$ 2$ we extend the concepts of semigroup and infinitesimal generator to an unbounded situation. Let $\left\{T_{t}\right\}_{t>0}$ be a one-parameter family of unbounded linear operators acting in a Banach space $X$, and suppose that for each element $x$ belonging to a suitable subspace $\mathbf{D}$ of $X, x \in \cap_{s, t>0} D\left(T_{s} T_{t}\right), T_{s} T_{t} x=T_{s+t} x$, $T_{t} x$ is a continuous function of $t>0$, and $\left\|T_{t} x-x\right\| \rightarrow 0$ as $t \rightarrow 0^{+}$.

In attempting to define the "infinitesimal generator" of $\left\{T_{t}\right\}_{t>0}$, one sees immediately that the operator $A x=\lim _{h \rightarrow 0^{+}}\left(\left(T_{h} x-x\right) / h\right)$, with domain $D(A)=\left\{x \in \cap_{t>0} D\left(T_{t}\right) \mid \lim _{h \rightarrow 0^{+}}\left(\left(T_{h} x-x\right) / h\right)\right.$ exists in $\left.X\right\}$ (in order to take limits we must work at least in $\bigcap_{t>0} D\left(T_{t}\right)$ ) fails to have the fundamental properties of the same operator in the classical setting. For example (even if we restrict our attention to the set $\mathbf{D})$, if $\lim _{h \rightarrow 0^{+}}\left(\left(T_{h} x-x\right) / h\right)$ exists, there is no guarantee that $\lim _{h \rightarrow 0^{+}}\left(\left(T_{h} T_{t} x-T_{t} x\right) / h\right)$ exists. Certainly further restrictions on the domain of $A$ are necessary if we wish to model our theory after the classical one; however it is not obvious at this point what these restrictions should be.

Our approach, inspired by the work of Feller [7] and [8], is via the Laplace transform; even for elements $x \in \mathrm{D}, \int_{0}^{\infty} e^{-\lambda t} T_{t} x d t, \lambda \in C$, need not converge. Thus we single out subspaces $\Sigma_{\omega}$ on which the Laplace transform of $T_{t} x$ is defined; for $\omega \in R$, let $N_{\omega}(x)=\sup _{t>0} e^{-\omega t}\left\|T_{t} x\right\|$, and set $\Sigma_{\omega}=\{x$ $\left.\in \mathbf{D} \mid N_{\omega}(x)<\infty\right\}$. Provided the semigroup $\left\{T_{t}\right\}_{t}>0$ satisfies a certain condition (which includes the case where each $T_{t}$ is closed), the spaces $\Sigma_{\omega}$ are Banach spaces with norm $N_{\omega}$ (Theorem 2.4); the operators defined by $J_{\lambda}^{\omega} x$ $=\int_{0}^{\infty} e^{-\lambda t} T_{t} x d t$, for $x \in \Sigma_{\omega}, \lambda \in C$ with $\operatorname{Re} \lambda>\omega$, are now bounded linear operators in $\left(\Sigma_{\omega}, N_{\omega}\right)$. In addition, they are one-to-one and satisfy the first resolvent equation; $A^{\omega}$ is defined to be the closed operator in $\left(\Sigma_{\omega}, N_{\omega}\right)$ whose resolvent is $J_{\lambda}^{\omega}$ (Theorem 2.9). Finally, the infinitesimal generator $A$ is defined so that the restriction of $A$ to each $\Sigma_{\omega}$ is $A^{\omega}$ (Definition 2.10). We are now in a position to decide what the additional restrictions on the domain of the operator $x \rightarrow \lim _{h \rightarrow 0^{+}}\left(\left(T_{h} x-x\right) / h\right)$ ought to be: $x \in D\left(A^{\omega}\right)$ if and only if $\lim _{h \rightarrow 0^{+}}\left(\left(T_{h} x-x\right) / h\right)$ exists in $X$, belongs to $\Sigma_{\omega}$, and $T_{t} x$ is a differentiable function of $t$ for $t>0$ (Theorem 2.15). Although the infinitesimal generator is not, in general, closed or densely defined, it does possess many of the properties of the classical generator, and in Corollaries 2.22 and 2.23 we obtain some of the classical representations of the semigroup $\left\{T_{t}\right\}_{t>0}$ on suitable subspaces of $X$. We also prove a "maximal-uniqueness" theorem (2.26), and Theorem 2.27 is a Hille-Yosida-type result in the spirit of the classical theorem.

In $\$ 3$ we illustrate our theory with concrete examples of semigroups of unbounded operators which occur in Banach space. First some properties of 
the Riemann-Liouville fractional integral acting in $L^{p}(0, \infty)$ are considered (Theorem 3.3); we then turn our attention to the closely related Weyl fractional integral, and obtain a one-parameter family of closed linear operators in $L^{p}(0, \infty), 1<p<\infty$, which provides a model for the theory developed in $\$ 2$. In Theorem 3.9 we prove that the infinitesimal generator of this semigroup is densely defined (this is not guaranteed by the general theory; cf. Remark 2.12).

We also consider suitably defined derivatives of fractional order in $L^{p}(0, \infty), 1<p<\infty$, and again obtain a semigroup of closed linear operators with densely defined infinitesimal generator (Theorem 3.12). Fractional derivatives in $L^{p}(0,1)$, defined as inverses of the Riemann-Liouville fractional integral, provide a counterexample to our theory; the infinitesimal generator in this case is the zero operator (Proposition 3.13).

Finally, we introduce fractional powers of closed operators, as defined by Balakrishnan [2]. These provide a wide class of examples which illustrate our theory; a simple sufficient condition that the domain of the infinitesimal generator be dense is given.

In a later paper (to appear) we will relate our results to those in [5] and [21] by applying our theory to semigroups of closed linear operators which satisfy certain additional restrictions; semigroups of unbounded scalar type operators with real spectrum are a special case (cf. [1] and [6, Vol. III, Chapter 18]).In this situation, the infinitesimal generator is a closable, densely defined operator which uniquely determines the semigroup.

Notation. We shall denote by $C$ the set of complex numbers and by $R$ the real numbers; $Z^{+}$will denote the set of positive integers. $X$ will be a nontrivial complex Banach space, and by a subspace of $X$ we shall mean any linear manifold in $X$.

For standard results in functional analysis and the classical theory of semigroups, see Hille and Phillips [12], Dunford and Schwartz [6], Kato [15] and Yosida [24].

2. The infinitesimal generator. Let $(X,\|\cdot\|)$ be a Banach space, and $\left\{T_{t}\right\}_{t>0}$ a one-parameter family of unbounded linear operators acting in $X$. Let $D\left(T_{t}\right)$ denote the domain of $T_{t}$, and set

$$
\mathbf{D}=\left\{\begin{array}{l|l}
x \in \underset{s, t>0}{\cap} D\left(T_{s} T_{t}\right) & \begin{array}{l}
T_{s} T_{t} x=T_{s+t} x, \\
T_{t} x \text { is strongly continuous for } t>0 \\
\left\|T_{t} x-x\right\| \rightarrow 0 \text { as } t \rightarrow 0^{+}
\end{array}
\end{array}\right\}
$$

If $\mathbf{D} \neq\{0\}$, we shall say that $\left\{T_{t}\right\}_{t>0}$ is a semigroup of unbounded operators in $X$. Definition 2.1. For $\omega \in R, x \in \cap_{t>0} D\left(T_{t}\right)$, let 


$$
N_{\omega}(x)=\sup _{t>0} e^{-\omega t}\left\|T_{t} x\right\|
$$

and set $\Sigma_{\omega}=\left\{x \in \mathbf{D} \mid N_{\omega}(x)<\infty\right\}$.

REMARK 2.2. (i) If $\left\{T_{t}\right\}_{t}>0$ is a semigroup of bounded operators of class $\left(C_{0}\right)$, then $\mathbf{D}=X$.

(ii) If for each $t>0, T_{t}$ is a closed operator in $X$, and $\mathbf{D}=X$, then $\left\{T_{t}\right\}_{t>0}$ is a semigroup of bounded operators of class $\left(C_{0}\right)$ on $X$ (closed graph theorem).

(iii) If $\left\{T_{t}\right\}_{t>0}$ is a semigroup of bounded operators of class $\left(C_{0}\right)$, then $X=\Sigma_{\omega}$ for any $\omega>\omega_{0}$, where

$$
\omega_{0}=\lim _{t \rightarrow \infty} \frac{1}{t} \log \left\|T_{t}\right\|
$$

is the type of $\left\{T_{t}\right\}_{t>0}$; moreover, $\|\cdot\|$ and $N_{\omega}(\cdot)$ are equivalent norms on $X$.

The following consequences of the definition are immediate:

Proposition 2.3. (i) For all $x \in \mathbf{D},\|x\| \leqslant N_{\omega}(x)$.

(ii) If $\omega_{1} \leqslant \omega_{2}$ and $x \in \mathbf{D}$, then $N_{\omega_{2}}(x) \leqslant N_{\omega_{1}}(x)$. Hence if $\omega_{1} \leqslant \omega_{2}$, then $\Sigma_{\omega_{1}} \subseteq \Sigma_{\omega_{2}}$.

(iii) If $x \in \Sigma_{\omega}^{\omega_{\omega}}$, then $T_{t} x \in \Sigma_{\omega}$ and $N_{\omega}\left(T_{t} x\right) \leqslant e^{\omega t} N_{\omega}(x)$.

For $\omega \in R$ and $t>0, T_{t} \mid \bar{\Sigma}_{\omega}$ will denote the operator $T_{t}$ with domain

$$
D\left(T_{t} \mid \bar{\Sigma}_{\omega}\right)=\left\{x \in \bar{\Sigma}_{\omega} \mid x \in D\left(T_{t}\right) \text { and } T_{t} x \in \bar{\Sigma}_{\omega}\right\}
$$

Here $\bar{\Sigma}_{\omega}$ denotes the closure of $\Sigma_{\omega}$ in the norm of $X$.

We now have the following

THEOREM 2.4. Let $\left\{T_{t}\right\}_{t>0}$ be a semigroup of unbounded linear operators in $X$. Let $\omega \in R$, and suppose that for each $t>0, T_{t} \mid \bar{\Sigma}_{\omega}$ is a closed operator in $\bar{\Sigma}_{\omega}$. Then $\Sigma_{\omega}$ is a Banach space, with norm $N_{\omega}$.

Proof. We emphasize that the operators $T_{t} \mid \bar{\Sigma}_{\omega}$ are closed with respect to the norm topology of $X$; clearly this condition implies that

$$
\begin{aligned}
& \text { if }\left\{x_{n}\right\} \subset \Sigma_{\omega},\left\|x_{n}-x\right\| \rightarrow 0 \text { and }\left\|T_{t} x_{n}-y\right\| \rightarrow 0, \\
& \text { then } x \in D\left(T_{t}\right) \text { and } T_{t} x=y .
\end{aligned}
$$

Now fix $\omega \in R$; it is clear that $N_{\omega}(\cdot)$ is a norm on $\Sigma_{\omega}$. Suppose $\left\{x_{n}\right\} \subset \Sigma_{\omega}$ is a Cauchy sequence with respect to the $N_{\omega}$-norm, and let $\varepsilon>0$. Then there exists a number $N$ such that for $m, n>N$,

$$
N_{\omega}\left(x_{m}-x_{n}\right)=\sup _{t>0} e^{-\omega t}\left\|T_{t} x_{m}-T_{t} x_{n}\right\|<\varepsilon .
$$


Thus $\left\|x_{m}-x_{n}\right\|<\varepsilon$ for $m, n>N$, by Proposition 2.3(i). Hence there exists $x \in X$ such that $\left\|x_{n}-x\right\| \rightarrow 0$ as $n \rightarrow \infty$. From (2.4.2) we see that, for each $t>0, m, n>N$,

$$
e^{-\omega t}\left\|T_{t} x_{m}-T_{t} x_{n}\right\|<\varepsilon
$$

that is, for each $t>0,\left\{e^{-\omega t} T_{t} x_{n}\right\}$ is a Cauchy sequence in the norm of $X$. So for each $t>0$ there exists $y_{t} \in X$ such that $\left\|e^{-\omega t} T_{t} x_{n}-y_{t}\right\| \rightarrow 0$ as $n \rightarrow \infty$. In (2.4.3) fix $n>N$, and let $m \rightarrow \infty$. Then

$$
\left\|e^{-\omega t} T_{t} x_{n}-y_{t}\right\| \leqslant \varepsilon
$$

where $N=N(\varepsilon)$ is independent of $t$.

Now, since $T_{t} \mid \bar{\Sigma}_{\omega}$ is closed for each $t>0$, so are the operators $\left(e^{-\omega t} T_{t} \mid \bar{\Sigma}_{\omega}\right)$, and (2.4.1) applied to $\left(e^{-\omega t} T_{t} \mid \bar{\Sigma}_{\omega}\right)$ implies $x \in D\left(e^{-\omega t} T_{t}\right)=D\left(T_{t}\right)$ and $e^{-\omega t} T_{t} x$ $=y_{t}$. By (2.4.4), we have that for $n>N, t>0$,

$$
e^{-\omega t}\left\|T_{t} x_{n}-T_{t} x\right\| \leqslant \varepsilon
$$

Since $N$ depends only on $\varepsilon, N_{\omega}\left(x_{n}-x\right) \leqslant \varepsilon$, for $n>N$. Therefore $N_{\omega}\left(x_{n}-x\right)$ $\rightarrow 0$ as $n \rightarrow \infty$. Consequently, $N_{\omega}(x)<\infty$. $\left(N_{\omega}(x)\right.$ is defined, for we have just shown that $x \in \cap_{t>0} D\left(T_{t}\right)$ ).

It remains to prove that $x \in \mathbf{D}$. Since $\left\{x_{n}\right\} \subset \mathbf{D},\left\{x_{n}\right\} \subset \cap_{s, t>0} D\left(T_{s} T_{t}\right)$, and $T_{s} T_{t} x_{n}=T_{s+t} x_{n}$ for all $s, t>0$, and each $n$. We now fix $s, t>0$. Then $N_{\omega}\left(x-x_{n}\right) \rightarrow 0$, so $\left\|T_{t} x_{n}-T_{t} x\right\| \rightarrow 0$ and $\left\|T_{s+t} x_{n}-T_{s+t} x\right\| \rightarrow 0$. But $T_{s+t} x_{n}$ $=T_{s} T_{t} x_{n}$, so that (2.4.1) may be applied again; thus $T_{t} x \in D\left(T_{s}\right)$, and $T_{s} T_{t} x$ $=T_{s+t} x$.

The strong continuity of $T_{t} x$ for $t>0$ follows from the fact that $e^{-\omega t} T_{t} x_{n}$ $\rightarrow e^{-\omega t} T_{t} x$ uniformly in $t$, as shown by (2.4.5).

Now for any $x \in \cap_{t>0} D\left(T_{t}\right),\left\|T_{t} x\right\| \leqslant e^{\omega t} N_{\omega}(x)$, so we have

$$
\left\|T_{t} x-x\right\| \leqslant e^{\omega t} N_{\omega}\left(x-x_{n}\right)+\left\|T_{t} x_{n}-x_{n}\right\|+N_{\omega}\left(x-x_{n}\right)
$$

For $n$ sufficiently large, choose $\delta>0$ such that if $0<t<\delta$, both $e^{\omega t}$ $<1+\varepsilon$ and $\left\|T_{t} x_{n}-x_{n}\right\|<\varepsilon$. Thus $\left\|T_{t} x-x\right\| \rightarrow 0$ as $t \rightarrow 0^{+}$, and hence $x \in \mathrm{D}$, as wanted.

Next, we show that the condition in Theorem 2.4 includes the case where the operators $T_{t}$ are closed.

Proposition 2.5. If $T_{t}$ is a closed operator in $X$, then $T_{t} \mid \bar{\Sigma}_{\omega}$ is closed in $\bar{\Sigma}_{\omega}$ for each $t>0, \omega \in R$.

Proof. $T_{t} \mid \bar{\Sigma}_{\omega} \subset T_{t}$, so if $T_{t}$ is closed, then $T_{t} \mid \bar{\Sigma}_{\omega}$ is closable, with closure $\widetilde{T}_{t} \mid \bar{\Sigma}_{\omega}$. If $x \in D\left(\widetilde{T_{t} \mid \bar{\Sigma}_{\omega}}\right)$, then there is a sequence $\left\{x_{n}\right\} \subset D\left(T_{t} \mid \bar{\Sigma}_{\omega}\right)$ such that 
$\left\|x_{n}-x\right\| \rightarrow 0$ and $\left\|T_{t} x_{n}-y\right\| \rightarrow 0$ for some $y \in \bar{\Sigma}_{\omega}$. Certainly $x \in \bar{\Sigma}_{\omega}$ and, since $T_{t}$ is closed, $x \in D\left(T_{t}\right)$ and $T_{t} x=y \in \bar{\Sigma}_{\omega}$. Thus $x \in D\left(T_{t} \mid \bar{\Sigma}_{\omega}\right)$, so that $T_{t} \mid \bar{\Sigma}_{\omega}$ is a closed operator in $\bar{\Sigma}_{\omega}$.

In the remainder of this section, all operators $T_{t}$ will satisfy the condition in Theorem 2.4.

Definition 2.6. For fixed $\omega \in R$, and $x \in \Sigma_{\omega}$, define

$$
J_{\lambda}^{\omega} x=\int_{0}^{\infty} e^{-\lambda t} T_{t} x d t, \quad \text { where } \lambda \in C, \operatorname{Re} \lambda>\omega .
$$

(Here the integral is an improper Riemann integral, converging absolutely in the norm of $X$.)

Observe that as an operator in $X, J_{\lambda}^{\omega}$ is in general unbounded, with domain $\Sigma_{\omega}$.

Lemma 2.7. Fix $\omega \in R$. Then for each $t>0, x \in \Sigma_{\omega}$ and $\lambda \in C$ with $\operatorname{Re} \lambda$ $>\omega$, we have $J_{\lambda}^{\omega} x \in D\left(T_{t}\right)$, and

$$
T_{t} J_{\lambda}^{\omega} x=J_{\lambda}^{\omega} T_{t} x
$$

Proof. Let $t>0$. Since $x \in \Sigma_{\omega}, T_{s} x \in D\left(T_{t}\right)$ for each $s>0$, and $T_{t} T_{s} x$ $\in \Sigma_{\omega}$. Since $T_{t} \mid \bar{\Sigma}_{\omega}$ is a closed operator in $\bar{\Sigma}_{\omega}$, we may apply Theorem III.6.20 in [6], and obtain $J_{\lambda}^{\omega} x \in D\left(T_{t}\right)$. Moreover,

$$
T_{t} J_{\lambda}^{\omega} x=T_{t} \int_{0}^{\infty} e^{-\lambda s} T_{s} x d s=\int_{0}^{\infty} e^{-\lambda s} T_{t} T_{s} x d s=J_{\lambda}^{\omega} T_{t} x
$$

THEOREM 2.8. For each $\omega \in R$ and $\lambda \in C$ with $\operatorname{Re} \lambda>\omega, J_{\lambda}^{\omega}\left(\Sigma_{\omega}\right) \subset \Sigma_{\omega}$. Moreover, the following hold:

(i) $N_{\omega}\left((\operatorname{Re} \lambda-\omega) J_{\lambda}^{\omega} x\right) \leqslant N_{\omega}(x)$ for each $x \in \Sigma_{\omega}$; hence $J_{\lambda}^{\omega}$ is a bounded operator on $\Sigma_{\omega}$.

(ii) For each $x \in \Sigma_{\omega},\left\|\lambda J_{\lambda}^{\omega} x-x\right\| \rightarrow 0$ as $\lambda \rightarrow \infty$.

(iii) $N_{\omega}(x)=\sup _{n \geqslant 0} \sup _{\lambda>\omega}\left\|(\lambda-\omega)^{n}\left(J_{\lambda}^{\omega}\right)^{n} x\right\|$, for each $x \in \Sigma_{\omega}$.

(iv) If $\omega_{1} \leqslant \omega_{2}, x \in \Sigma_{\omega_{1}}$ and $\operatorname{Re} \lambda>\omega_{2}$, then $J_{\lambda}^{\omega_{1}} x=J_{\lambda}^{\omega_{2}} x$. Thus as operators in $X, J_{\lambda}^{\omega_{1}} \subset J_{\lambda}^{\omega_{2}}$ if $\operatorname{Re}>\omega_{2}$.

Proof. To prove the first statement, fix $\omega \in R$; let $x \in \Sigma_{\omega}$ and $\lambda$ $\in C$ with $\operatorname{Re} \lambda>\omega$. We must show that $J_{\lambda}^{\omega} x \in \mathbf{D}$, and $N_{\omega}\left(J_{\lambda}^{\omega} x\right)<\infty$. From Lemma 2.7 we have that $J_{\lambda}^{\omega} x \in \cap_{t>0} D\left(T_{t}\right)$, and $T_{t} J_{\lambda}^{\omega} x=J_{\lambda}^{\omega} T_{t} x$. Similarly, we obtain $J_{\lambda}^{\omega} x \in \cap_{s, t>0} D\left(T_{s} T_{t}\right)$, and $T_{s} T_{t} J_{\lambda}^{\omega} x=T_{s+l} J_{\lambda}^{\omega} x$, for all $s, t>0$.

Now for any $h>0$, we have $T_{h} J_{\lambda}^{\omega} x=\int_{0}^{\infty} e^{-\lambda s} T_{s+h} x d s$. Since $x \in \Sigma_{\omega}$, for each $s>0,\left\|T_{s+h} x-T_{s+t} x\right\| \rightarrow 0$ as $h \rightarrow t$. Moreover, $\left\|T_{s+h} x\right\|$ $\leqslant e^{\omega(s+h)} N_{\omega}(x) \leqslant M_{t} e^{\omega s} N_{\omega}(x)$ provided $|t-h|$ is sufficiently small (here $M_{t}$ is 
a constant depending on $t$ ). Thus by the dominated convergence theorem (for vector-valued integrals, cf. [6, Chapter III]), $\left\|T_{h} J_{\lambda}^{\omega} x-T_{t} J_{\lambda}^{\omega} x\right\| \rightarrow 0$ as $|t-h|$ $\rightarrow 0$. By a similar argument, $\left\|T_{t} J_{\lambda}^{\omega} x-J_{\lambda}^{\omega} x\right\| \rightarrow 0$ as $t \rightarrow 0^{+}$. Hence $J_{\lambda}^{\omega} x$ $\in \mathbf{D}$; for each $t>0$,

$$
e^{-\omega t}\left\|T_{t} J_{\lambda}^{\omega} x\right\| \leqslant N_{\omega}(x) \int_{0}^{\infty} e^{(\omega-\operatorname{Re} \lambda) s} d s<\infty,
$$

provided $\operatorname{Re} \lambda>\omega$. Since the right-hand side of (2.8.1) is independent of $t$, it follows that $N_{\omega}\left(J_{\lambda}^{\omega} x\right)<\infty$. Thus $x \in \Sigma_{\omega}$, as wanted.

(i) follows immediately from (2.8.1), by miltiplying both sides of the inequality by $(\operatorname{Re} \lambda-\omega)$. This also shows that $J_{\lambda}^{\omega}$ is a bounded operator on $\Sigma_{\omega}$; linearity is clear.

To prove (ii), let $\varepsilon>0$ and choose $\delta>0$ so that, for $0<t<\delta,\left\|T_{t} x-x\right\|$ $<\varepsilon$. Then for $\lambda>\omega$,

$$
\begin{aligned}
\left\|\lambda J_{\lambda} x-x\right\| & \leqslant \lambda \int_{0}^{\infty} e^{-\lambda t}\left\|T_{t} x-x\right\| d t \\
& <\varepsilon \lambda \int_{0}^{\delta} e^{-\lambda t} d t+\lambda \int_{\delta}^{\infty} e^{-\lambda t}\left(e^{\omega t} N_{\omega}(x)+\|x\|\right) d t \\
& =\left(1-e^{-\lambda \delta}\right) \varepsilon+\frac{\lambda}{\lambda-\omega} e^{(\omega-\lambda) \delta} N_{\omega}(x)+e^{-\lambda \delta}\|x\| \rightarrow \varepsilon \quad \text { as } \lambda \rightarrow \infty .
\end{aligned}
$$

Since $\varepsilon$ was arbitrary, (ii) is proved.

The proof of (iii) is essentially the same as that in [8]; we present the details for completeness. By induction, we show that for $x \in \Sigma_{\omega}, \operatorname{Re} \lambda>\omega$ and $n$ $\geqslant 1$,

$$
\left(J_{\lambda}^{\omega}\right)^{n} x=\frac{1}{(n-1) !} \int_{0}^{\infty} e^{-\lambda t} t^{n-1} T_{t} x d t .
$$

$\left(J_{\lambda}^{\omega}\right)^{n} x$ is well defined, as we have just shown that $J_{\lambda}^{\omega}\left(\Sigma_{\omega}\right) \subset \Sigma_{\omega}$. For $n=1$, (2.8.2) is the definition of $J_{\lambda}^{\omega}$; so assume it holds for $n=k$. Then

$$
\begin{aligned}
\left(J_{\lambda}^{\omega}\right)^{k+1} x & =J_{\lambda}^{\omega}\left[\frac{1}{(k-1) !} \int_{0}^{\infty} e^{-\lambda t} t^{k-1} T_{t} x d t\right] \\
& =\frac{1}{(k-1) !} \int_{0}^{\infty} e^{-\lambda s} T_{s}\left[\int_{0}^{\infty} e^{-\lambda t} t^{k-1} T_{t} x d t\right] d s \\
& =\frac{1}{(k-1) !} \int_{0}^{\infty} e^{-\lambda s} \int_{0}^{\infty} e^{-\lambda t} t^{k-1} T_{s+t} x d t d s,
\end{aligned}
$$

again using Lemma 2.7. Changing variables and interchanging the order of integration, we get 


$$
\begin{aligned}
\left(J_{\lambda}^{\omega}\right)^{k+1} x & =\frac{1}{(k-1) !} \int_{0}^{\infty} e^{-\lambda s} \int_{s}^{\infty} e^{-\lambda(u-s)}(u-s)^{k-1} T_{u} x d u d s \\
& =\frac{1}{(k-1) !} \int_{0}^{\infty} e^{-\lambda u} T_{u} x \int_{0}^{u}(u-s)^{k-1} d s d u \\
& =\frac{1}{k !} \int_{0}^{\infty} e^{-\lambda u} u^{k} T_{u} x d u .
\end{aligned}
$$

Here we use the Fubini theorem, since $\left\|T_{u} x\right\| \leqslant e^{u \omega} N_{\omega}(x)$ implies that the iterated integral (2.8.3) converges absolutely.

Now from (2.8.2) we see that the right-hand side of (iii) is dominated by $N_{\omega}(x)$. For the other inequality, we use the following (cf. [8]):

If $f(t)$ is continuous and bounded, and if $\lambda \rightarrow \infty, n \rightarrow \infty$ so that

$$
(n-1) /(\lambda-\omega) \rightarrow t
$$

then

$$
\frac{(\lambda-\omega)^{n}}{(n-1) !} \int_{0}^{\infty} e^{-(\lambda-\omega) s} s^{n-1} f(s) d s \rightarrow f(t) .
$$

We apply this to the bounded, continuous function $f(t)=e^{-\omega t} T_{t} x$, where $x$ $\in \Sigma_{\omega}$. Then for all $t>0$

$$
\begin{aligned}
e^{-\omega t}\left\|T_{t} x\right\| & \leqslant \lim \sup \left\|(\lambda-\omega)^{n}\left(J_{\lambda}^{\omega}\right)^{n} x\right\| \\
& \leqslant \sup _{n \geqslant 0} \sup _{\lambda>\omega}\left\|(\lambda-\omega)^{n}\left(J_{\lambda}^{\omega}\right)^{n} x\right\|,
\end{aligned}
$$

and so (iii) is proved.

The next theorem will enable us to define the "infinitesimal generator" of the semigroup $\left\{T_{t}\right\}_{t>0}$.

THEOREM 2.9. For each $\omega \in R$, the family of operators $\left[J_{\lambda}^{\omega}: \operatorname{Re} \lambda>\omega\right]$ on $\Sigma_{\omega}$ is the resolvent of a closed linear operator $A^{\omega}$ in $\Sigma_{\omega}$ (i.e. closed in the $N_{\omega}$-norm topology).

Proof. Fix $\omega \in R$. We show that the operators $\left[J_{\lambda}^{\omega}: \operatorname{Re} \lambda>\omega\right]$ satisfy the first resolvent equation. Indeed, let $\lambda, \mu \in C, \lambda \neq \mu, \operatorname{Re} \lambda, \operatorname{Re} \mu>\omega$. Then for $x \in \Sigma_{\omega}, J_{\lambda}^{\omega} J_{\mu}^{\omega} x$ is well defined; using Lemma 2.7 and changing variables,

$$
\begin{aligned}
J_{\lambda}^{\omega} J_{\mu}^{\omega} x & =\int_{0}^{\infty} e^{-\lambda s} \int_{0}^{\infty} e^{-\mu t} T_{s+t} x d t d s=\int_{0}^{\infty} e^{-(\lambda-\mu) s} \int_{0}^{\infty} e^{-\mu(t+s)} T_{s+t} x d t d s \\
& =\int_{0}^{\infty} e^{-(\lambda-\mu) s} \int_{s}^{\infty} e^{-\mu u} T_{u} x d u d s=\int_{0}^{\infty} e^{-\mu u} T_{u} x \int_{0}^{u} e^{-(\lambda-\mu) s} d s d u \\
& =\int_{0}^{\infty} e^{-\mu u} T_{u} x\left[\frac{-e^{-(\lambda-\mu) u}}{\lambda-\mu}+\frac{1}{\lambda-\mu}\right] d u=\frac{1}{\lambda-\mu}\left[J_{\mu}^{\omega} x-J_{\lambda}^{\omega} x\right]
\end{aligned}
$$


where Fubini's theorem again permits the interchange of order of integration, since $\left\|T_{s+t} x\right\| \leqslant e^{\omega(s+t)} N_{\omega}(x)$.

Next, we prove that $J_{\lambda}^{\omega}$ is 1-1 on $\Sigma_{\omega^{*}}$. For suppose $J_{\lambda}^{\omega} x=0$ for $x \in \Sigma_{\omega}, \lambda$ $\in C$ with $\operatorname{Re} \omega>\lambda$. Then for $\mu \in C$ with $\operatorname{Re} \mu>\omega, \mu \neq \lambda, J_{\mu}^{\omega}\left(J_{\lambda}^{\omega} x\right)=0$, so we see from (2.9.1) that $J_{\mu}^{\omega} x=0$ for all $\mu \in C$ with $\operatorname{Re} \mu>\omega$. Thus the uniqueness theorem for Laplace transforms (cf. [12, Theorem 6.2.3]) implies $T_{t} x=0$ for each $t>0$. But $x \in \Sigma_{\omega}$, so $\left\|T_{t} x-x\right\| \rightarrow 0$ as $t \rightarrow 0^{+}$. Therefore $x=0$. Now by Theorem 5.8.3 in [12], $\left[J_{\lambda}^{\omega}: \operatorname{Re} \lambda>\omega\right]$ is the resolvent of a closed linear operator $A^{\omega}$ in the Banach space $\left(\Sigma_{\omega}, N_{\omega}\right)$, and $A^{\omega}=\lambda I$ $-\left(J_{\lambda}^{\omega}\right)^{-1}$, for $\lambda \in C$ with $\operatorname{Re} \lambda>\omega$; also, Domain $\left(A^{\omega}\right)=\operatorname{Range}\left(J_{\lambda}^{\omega}\right)$. (Note that the definition of $A^{\omega}$ is independent of the choice of $\lambda$, and that Range $\left(J_{\lambda_{1}}^{\omega}\right)=\operatorname{Range}\left(J_{\lambda_{2}}^{\omega}\right)$ for $\lambda_{1}, \lambda_{2} \in C$ with $\operatorname{Re} \lambda_{1}, \operatorname{Re} \lambda_{2}>\omega$, again by (2.9.1).)

The operators $A^{\omega}$ are not, in general, closed in the norm of $X$.

Definition 2.10. Let $D(A)=\cup_{\omega \in R} D\left(A^{\omega}\right)$; for $x \in D(A)$, define $A x=\lambda x$ $-y$ if $x \in D\left(A^{\omega}\right)$ and $x=J_{\lambda}^{\omega} y$ for $y \in \Sigma_{\omega}, \operatorname{Re} \lambda>\omega$. We call $A$ the infinitesimal generator of the semigroup $\left\{T_{t}\right\}_{t>0}$.

Note. A is a well defined (cf. Theorem 2.11(i)), and the restriction of $A$ to $\Sigma_{\omega}\left(\right.$ denoted $\left.A \mid \Sigma_{\omega}\right)$, with domain

$$
D\left(A \mid \Sigma_{\omega}\right)=\left\{x \in \Sigma_{\omega} \mid x \in D(A) \text { and } A x \in \Sigma_{\omega}\right\},
$$

is simply the operator $A^{\omega}$. Indeed, that $A^{\omega} \subset A \mid \Sigma_{\omega}$ is clear. On the other hand, if $x \in D\left(A \mid \Sigma_{\omega}\right)$, then $x \in D\left(A^{\omega^{\prime}}\right)$ for some $\omega^{\prime} \in R$, so $x=J_{\lambda}^{\omega^{\prime}} y$ for $y \in \Sigma_{\omega^{\prime}}$; we may choose $\lambda \in C$ with $\operatorname{Re} \lambda>\max \left(\omega^{\prime}, \omega\right)$. Thus $A x=A^{\omega^{\prime}} x$ $=\lambda x-y$. But $A x \in \Sigma_{\omega}$, by assumption, so $y \in \Sigma_{\omega}$ and therefore $x$ $\in D\left(A^{\omega}\right)$.

TheOREM 2.11. (i) $A$ is well defined. In fact, if $\omega_{1} \leqslant \omega_{2}$ then $A^{\omega_{1}} \subset A^{\omega_{2}}$.

(ii) $D(A)$ is a linear manifold and $A$ is a linear operator in $X$.

(iii) For $x \in \Sigma_{\omega}$, the resolvent equation

$$
(\lambda I-A) y=x, \quad \operatorname{Re} \lambda>\omega,
$$

has a unique solution $y$ belonging to $\Sigma_{\omega}$, and $y=J_{\lambda}^{\omega} x$.

(iv) For each $\omega \in R, \overline{D\left(A^{\omega}\right)}=\bar{\Sigma}_{\omega}$ and $\overline{D(A)}=\bar{\Sigma}$, where $\Sigma=\cup_{\omega \in R} \Sigma_{\omega}$. (Here the closures are taken in the norm of $X$.)

(v) Let $\omega \in R$. Then for each $t>0, T_{t}\left(D\left(A^{\omega}\right)\right) \subset D\left(A^{\omega}\right)$, and

$$
T_{t} A^{\omega} x=A^{\omega} T_{t} x
$$

Proof. (i) Suppose $x \in D\left(A^{\omega_{1}}\right)$, and $\omega_{2} \geqslant \omega_{1}$. Since $D\left(A^{\omega_{1}}\right)$ $=$ Range $\left(J_{\lambda}^{\omega_{1}}\right), x=J_{\lambda}^{\omega_{1}} y$ for some $y \in \Sigma_{\omega_{1}}$ and $\lambda \in C$ with $\operatorname{Re} \lambda>\omega_{2}$; this 
is possible because the set Range $\left(J_{\lambda}^{\omega_{1}}\right)$ does not depend on $\lambda$. Then $A x$ $=A^{\omega_{1}} x=\lambda x-y$. But $J_{\lambda}^{\omega_{1}} y=J_{\lambda}^{\omega_{2}} y$, by Theorem 2.8(iv). Thus $x$ $\in$ Range $\left(J_{\lambda}^{\omega_{2}}\right)=D\left(A^{\omega_{2}}\right)$, and $A x=A^{\omega_{2}} x=\lambda x-y$. Therefore if $x$ $\in D(A)$, then $A x=A^{\omega} x$ for any $\omega \in R$ for which $x \in D\left(A^{\omega}\right)$, and so $A$ is well defined. We have also shown that if $\omega_{1} \leqslant \omega_{2}$, then $A^{\omega_{1}} \subset A^{\omega_{2}}$ as operators in $X$.

(ii) is now clear, since the sets $D\left(A^{\omega}\right), \omega \in R$, are nested.

(iii) Fix $x \in \Sigma_{\omega}$, and $\lambda \in C$ with $\operatorname{Re} \lambda>\omega$; certainly $y=J_{\lambda}^{\omega} x$ is a solution of (2.11.1), and $J_{\lambda}^{\omega} x \in \Sigma_{\omega}$. That $J_{\lambda}^{\omega} x$ is the unique solution belonging to the set $\Sigma_{\omega}$ follows from the fact that $J_{\lambda}^{\omega}=R\left(\lambda ; A^{\omega}\right)$ for $\operatorname{Re} \lambda>\omega$, and $A \mid \Sigma_{\omega}=A^{\omega}$.

(iv) $\overline{D\left(A^{\omega}\right)}=\bar{\Sigma}_{\omega}$ follows from (ii) of Theorem 2.8, for $J_{\lambda}^{\omega} x \in D\left(A^{\omega}\right)$ whenever $x \in \Sigma_{\omega}, \operatorname{Re} \lambda>\omega$. Thus

$$
\begin{aligned}
\bar{\Sigma} & =\overline{\bigcup_{\omega} \Sigma_{\omega}} \subset \overline{U \bar{\Sigma}_{\omega}}=\overline{\bigcup_{\omega} \overline{D\left(A^{\omega}\right)}} \subset \overline{\bigcup_{\omega} D\left(A^{\omega}\right)} \\
& =\overline{D(A)} \subset \overline{\bigcup_{\omega} \Sigma_{\omega}}=\bar{\Sigma} .
\end{aligned}
$$

(v) Let $x \in D\left(A^{\omega}\right)$. Then $x=J_{\lambda}^{\omega} y=\int_{0}^{\infty} e^{-\lambda s} T_{s} y d s$ for some $y \in \Sigma_{\omega}$, $\operatorname{Re} \lambda>\omega$. By Lemma 2.7, $T_{t} J_{\lambda}^{\omega} y=J_{\lambda}^{\omega} T_{t} y \in D\left(A^{\omega}\right)$, and so

$$
A^{\omega} T_{t} x=A^{\omega} T_{t} J_{\lambda}^{\omega} y=A^{\omega} J_{\lambda}^{\omega} T_{t} y=\lambda T_{t} x-T_{t} y .
$$

Moreover, since $A^{\omega} x=\lambda x-y$, we have

$$
T_{t} A^{\omega} x=\lambda T_{t} x-T_{t} y=A^{\omega} T_{t} x,
$$

as wanted.

Note that if $\left\{T_{t}\right\}_{t}>0$ is a semigroup of bounded operators of class $\left(C_{0}\right)$ on $X$, then for sufficiently large $\omega \in R$ (cf. Remark 2.2 (iii)), $A=A^{\omega}$ is the classical infinitesimal generator; moreover,

$$
J_{\lambda}^{\omega} x=R(\lambda ; A) x=\int_{0}^{\infty} e^{-\lambda t} T_{t} x d t, \quad \operatorname{Re} \lambda>\omega, \quad x \in X .
$$

REMARK 2.12. Given the generality of our theory as developed so far, we have no guarantee that $A$ is densely defined. Of course, (iv) of Theorem 2.11 shows that if $\bar{\Sigma}=X$, then $D(A)$ is dense in $X$. However, in $\S 3$ we shall give several examples for which the generator is in fact densely defined.

Next, we show that the infinitesimal generator possesses many of the desirable properties of the classically defined operator.

THEOREM 2.13. (i) For $x \in D(A), \lim _{h \rightarrow 0^{+}}\left(\left(T_{h} x-x\right) / h\right)$ exists in the norm of $X$, and is equal to $A X$. 
(ii) If $x \in D(A)$, then $T_{t} x \in D(A)$ and $T_{t} x$ is a differentiable function of $t$ for $t>0$, with $d T_{t} x / d t=T_{t} A x=A T_{t} x$. Hence for each $x \in D(A), T_{t} x-x$ $=\int_{0}^{t} T_{s} A x d s$.

Proof. (i) Since $A x=A^{\omega} x$ if $x \in D\left(A^{\omega}\right)$, we fix an $\omega \in R$ and prove the result for $A^{\omega}$. Thus if $x \in D\left(A^{\omega}\right)$, then $x=J_{\lambda}^{\omega} y$ for some $y$ $\in \Sigma_{\omega}$ and $\operatorname{Re} \lambda>\omega$, and $A^{\omega} x=\lambda x-y$. Then for $h>0$,

$$
\frac{T_{h} x-x}{h}=\frac{e^{\lambda h}-1}{h} \int_{0}^{\infty} e^{-\lambda t} T_{t} y d t-\frac{e^{\lambda h}}{h} \int_{0}^{h} e^{-\lambda t} T_{t} y d t .
$$

Thus

$$
\lim _{h \rightarrow 0^{+}} \frac{T_{h} x-x}{h}=\lambda x-y=A^{\omega} x,
$$

where the last expression in (2.13.1) converges in the $X$-norm to $y$ since $\left\|T_{t} y-y\right\| \rightarrow 0$ as $t \rightarrow 0^{+}$.

(ii) (cf. [24, p. 239]). Suppose $x \in D\left(A^{\omega}\right)$ for $\omega \in R$. Then we have already shown in (v) of Theorem 2.11 that $T_{t} x \in D\left(A^{\omega}\right) \subset D(A)$, and $T_{t} A x=T_{t} A^{\omega} x$ $=A^{\omega} T_{t} x=A T_{t}$.

Now let $x \in D\left(A^{\omega}\right)$, and $x^{*} \in X^{*}$, the conjugate space of $X$. Then $f(t)=x^{*} T_{t} x$ is a continuous function of $t, t>0$; from (i) applied to $T_{t} x$, we see that $f(t)$ possesses a right derivative $x^{*} A^{\omega} T_{t} x=x^{*} T_{t} A^{\omega} x$. Since $A^{\omega} x$ $\in \Sigma_{\omega}, x^{*} T_{t} A^{\omega} x$ is continuous and finite as a function of $t$. But if one of the four Dini derivates of $f(t)$ is finite and continuous, $f(t)$ is differentiable, and $d\left(x^{*} T_{t} x\right) / d t=x^{*} A^{\omega} T_{t} x=x^{*} T_{t} A^{\omega} x$. Thus $x^{*}\left(T_{t} x-x\right)=\int_{0}^{t} x^{*} T_{s} A^{\omega} x d s$. Since $x^{*} \in X^{*}$ was chosen arbitrarily,

$$
T_{t} x-x=\int_{0}^{t} T_{s} A^{\omega} x d s=\int_{0}^{t} T_{s} A x d s=\int_{0}^{t} A T_{s} x d s .
$$

Thus $T_{t} x$ is differentiable as a function of $t$, and the derivative has the desired value.

Definition 2.14. Let $\omega \in R$, and set

$$
D\left(A_{0}^{\omega}\right)=\left\{\begin{array}{l|l}
x \in \Sigma_{\omega} & \begin{array}{l}
\exists y \in \Sigma_{\omega} \text { such that }\left\|\left(\left(T_{t} x-x\right) / t\right)-y\right\| \rightarrow 0 \\
\text { as } t \rightarrow 0^{+}, \text {and } T_{t} x \text { is differentiable } \\
\text { for } t>0
\end{array}
\end{array}\right\}
$$

For $x \in D\left(A_{0}^{\omega}\right)$, define

$$
A_{0}^{\omega} x=\lim _{t \rightarrow 0^{+}} \frac{T_{t} x-x}{t} .
$$

Thus $A_{0}^{\omega}$ is an operator from $\Sigma_{\omega}$ into itself, with domain $D\left(A_{0}^{\omega}\right)$. Let $D\left(A_{0}\right)=\cup D\left(A_{0}^{\omega}\right)$, and define $A_{0} x=A_{0}^{\omega} x$ if $x \in D\left(A_{0}^{\omega}\right)$. Then $A_{0}$ is well 
defined, and $A_{0}^{\omega}=A_{0} \mid \Sigma_{\omega}$, where $A_{0} \mid \Sigma_{\omega}$ is defined as usual.

THEOREM 2.15. Let $A$ be the infinitesimal generator of the semigroup of unbounded operators $\left\{T_{t}\right\}_{t>0}$. Then $A=A_{0}$.

Proof. We show that for each $\omega \in R, A^{\omega}=A_{0}^{\omega}$; it suffices to prove that $J_{\lambda}^{\omega}=R\left(\lambda ; A^{\omega}\right)$ is also the resolvent of the operator $A_{0}^{\omega}$ acting in the Banach space $\Sigma_{\omega}$.

By Theorem 2.13, we have that $D\left(A^{\omega}\right) \subset D\left(A_{0}^{\omega}\right)$, for if $x \in D\left(A^{\omega}\right), A^{\omega} x$ $=\lim _{t \rightarrow 0^{+}}\left(\left(T_{t} x-x\right) / t\right)$ belongs to $\Sigma_{\omega}$, and $T_{t} x$ is differentiable; also, $A^{\omega} x$ $=A_{0}^{\omega} x$. Now if $x \in \Sigma_{\omega}$, then $J_{\lambda}^{\omega} x \in D\left(A^{\omega}\right) \subset D\left(A_{0}^{\omega}\right)$, so that for $\operatorname{Re} \lambda>\omega$,

$$
\left(\lambda I-A_{0}^{\omega}\right) J_{\lambda}^{\omega} x=\left(\lambda I-A^{\omega}\right) J_{\lambda}^{\omega} x=x .
$$

We claim that $J_{\lambda}^{\omega} x$ is the unique solution of the resolvent equation

$$
\left(\lambda I-A_{0}^{\omega}\right) y=x,
$$

where $x \in \Sigma_{\omega}, \operatorname{Re} \lambda>\omega$, in which case $J_{\lambda}^{\omega}$ will be the resolvent of $A_{0}^{\omega}$. Indeed, suppose $\lambda y-A_{0}^{\omega} y=0$ for some $y \in D\left(A_{0}^{\omega}\right)$. Then for $t>0, \lambda T_{t} y$ - $T_{t} A_{0}^{\omega} y=0$, where $T_{t} A_{0}^{\omega} y$ is defined since $A_{0}^{\omega} y \in \Sigma_{\omega}$. But $y \in D\left(A_{0}^{\omega}\right)$, so that $T_{t} y$ is differentiable for $t>0$, and $\left\|T_{t} y-y\right\| \rightarrow 0$ as $t \rightarrow 0^{+}$. Moreover, $d T_{t} y / d t=T_{t} A_{0}^{\omega} y=\lambda T_{t} y$, for

$$
\lim _{h \rightarrow 0^{+}} \frac{T_{t+h} y-T_{t} y}{h}=\lim _{h \rightarrow 0^{+}} T_{t}\left(\frac{T_{h} y-y}{h}\right)
$$

and

$$
A_{0}^{\omega} y=\lim _{h \rightarrow 0^{+}} \frac{T_{h} y-y}{h} .
$$

Since we are assuming that $T_{t} \mid \bar{\Sigma}_{\omega}$ is a closed operator in $\bar{\Sigma}_{\omega}, A_{0}^{\omega} y \in D\left(T_{t}\right)$ and $d T_{t} y / d t=T_{t} A_{0}^{\omega} y$, as claimed. Thus $T_{t} y=e^{\lambda t} y$ for fixed $\lambda \in C$ with $\operatorname{Re} \lambda$ $>\omega$. But this can be the case only if $y=0$, since $N_{\omega}(y)$ $=\sup _{t>0} e^{-(\omega-\operatorname{Re} \lambda) t}\|y\|<\infty$. Therefore $J_{\lambda}^{\omega}=R\left(\lambda ; A_{0}^{\omega}\right)$; hence $A_{0}^{\omega}=A^{\omega}$.

Theorem 2.15 enables us to redefine our generator $A$ in a manner more compatible with the classical definition. The definition of $A$ given by 2.14 does not depend on the completeness of $\Sigma_{\omega}$ with respect to the norm $N_{\omega}$; thus the infinitesimal generator may be defined without any additional hypothesis on the semigroup $\left\{T_{t}\right\}_{t>0}$. However, if we take Definition 2.14 as a starting point, and add the condition that the operators $T_{t} \mid \bar{\Sigma}_{\omega}$ are closed in $\bar{\Sigma}_{\omega}$, then we obtain the operators $A^{\omega}$ in their original form. Indeed, in Theorem 2.15 we proved that $R\left(\lambda ; A_{0}^{\omega}\right)=J_{\lambda}^{\omega}$. Thus we have the following

THEOREM 2.16. Let $\left\{T_{t}\right\}_{t>0}$ be a semigroup of unbounded linear operators in $X$, and suppose that for each $\omega \in R, t>0, T_{t} \mid \bar{\Sigma}_{\omega}$ is a closed operator in $\bar{\Sigma}_{\omega}$. Let $A_{0}$ 
be the operator defined in 2.14. Then $\left(\Sigma_{\omega}, N_{\omega}\right)$ is a Banach space, and for each $x \in \Sigma_{\omega}$ and $\lambda \in C$ with $\operatorname{Re} \lambda>\omega$, the resolvent equation

$$
\left(\lambda I-A_{0}\right) y=x
$$

has a unique solution, say $y=J_{\lambda}^{\omega} x$, where $y \in \Sigma_{\omega}$ and

$$
J_{\lambda}^{\omega} x=\int_{0}^{\infty} e^{-\lambda t} T_{t} x d t
$$

Moreover, the operator $J_{\lambda}^{\omega}: \Sigma_{\omega} \rightarrow \Sigma_{\omega}$ has properties (i)-(iv) of Theorem 2.8 .

Next we prove that the infinitesimal generator of a semigroup of unbounded operators is, in a certain sense, unique (Theorem 2.26). We begin with

Lemma 2.17. Let $X$ be a Banach space, $A$ and $B$ linear operators in $X$ with domains $D(A), D(B)$, respectively. Suppose that

(i) $A \subset B$,

(ii) $\rho(A) \cap \rho(B) \neq \varnothing$.

Then $A=B$.

Proof. In view of (i), it suffices to prove that $D(A)=D(B)$. Let $\lambda$ $\in \rho(A) \cap \rho(B)$. Then $(\lambda I-B)$ maps $D(B) 1-1$ and onto $X$. In addition, $R(\lambda ; A)$ exists, and for each $x \in X$,

$$
(\lambda I-B) R(\lambda ; A) x=(\lambda I-A) R(\lambda ; A) x=x,
$$

since $R(\lambda ; A) x \in D(A) \subset D(B)$. Thus $(\lambda I-B)$ maps $D(A)$ onto $X$ in a 1-1 fashion.

Hence $(\lambda I-B)$ maps both $D(A)$ and $D(B) 1-1$ and onto $X$. Since $D(A) \subset D(B)$, it must be the case that $D(A)=D(B)$.

Throughout this section, $A$ will denote the infinitesimal generator of $\left\{T_{t}\right\}_{t>0}$, and $A^{\omega}=A \mid \Sigma_{\omega}$, where $\Sigma_{\omega}$ was defined in (2.1).

Definition 2.18. For each $\omega \in R$, let

$$
\mathscr{D}_{\omega}=\overline{D\left(A^{\omega}\right)} N_{\omega}
$$

(i.e. closure in the $N_{\omega}$-norm; note that $\mathscr{D}_{\omega} \subset \Sigma_{\omega}$ ). Then $A^{\omega} \mid \mathscr{D}_{\omega}$ will denote the operator $A^{\omega}$ with domain

$$
D\left(A^{\omega} \mid \mathscr{D}_{\omega}\right)=\left\{x \in \mathscr{D}_{\omega} \mid x \in D\left(A^{\omega}\right) \text { and } A^{\omega} x \in \mathscr{D}_{\omega}\right\} .
$$

Remark 2.19. Since $A^{\omega}$ is a closed operator in the Banach space $\left(\Sigma_{\omega}, N_{\omega}\right)$, we have by Theorem 12.2.4 in [12] that $A^{\omega} \mid \mathscr{D}_{\omega}$ is a closed operator in $\mathscr{D}_{\omega}$, with $\overline{D\left(A^{\omega} \mid \mathscr{D}_{\omega}\right)} N_{\omega}=\mathscr{D}_{\omega}$. Moreover, for all $\lambda \in \rho\left(A^{\omega}\right)$, 


$$
R\left(\lambda ; A^{\omega} \mid \mathscr{D}_{\omega}\right)=\left[R\left(\lambda ; A^{\omega}\right)\right] \mid \mathscr{D}_{\omega},
$$

the restriction of $R\left(\lambda ; A^{\omega}\right)$ to the (invariant) subspace $\mathscr{D}_{\omega}$.

In light of this remark, we have

THEOREM 2.20. Let $\omega \in R$. Then for each $t>0, T_{t} \mathscr{D}_{\omega} \subset \mathscr{D}_{\omega}$ and $\left\{T_{t} \mid \Phi_{\omega}\right\}$ (denoted $T_{t}^{\omega}$ ) is a semigroup of bounded linear operators of class $\left(C_{0}\right)$ on the Banach space $\left(\mathscr{D}_{\omega}, N_{\omega}\right)$, with infinitesimal generator (in the classical sense) $A^{\omega} \mid \mathscr{D}_{\omega}$.

Proof. Fix $t>0$. That $T_{t} \mathscr{D}_{\omega} \subset \mathscr{D}_{\omega}$ is clear, since $T_{t}$ is a bounded operator on $\left(\Sigma_{\omega}, N_{\omega}\right)$, and $T_{t}\left(D\left(A^{\omega}\right)\right) \subset D\left(A^{\omega}\right)$ (Theorem 2.11(v)).

Let $x \in D\left(A^{\omega}\right)$. Then by Theorem 2.13(ii), $T_{t} x-x=\int_{0}^{t} T_{s} A x d s$. So for $h>0$,

$$
\begin{aligned}
e^{-\omega h}\left\|T_{t+h} x-T_{h} x\right\| & \left.\leqslant t e^{-\omega h}\left\|T_{\xi+h} A x\right\| \quad \text { (where } 0<\xi<t\right) \\
& \leqslant t e^{\omega \xi} N_{\omega}(A x) \rightarrow 0 \quad \text { as } t \rightarrow 0^{+},
\end{aligned}
$$

uniformly in $h$. Therefore $N_{\omega}\left(T_{t} x-x\right) \rightarrow 0$ as $t \rightarrow 0^{+}$; similarly, $T_{t} x$ is strongly continuous in the $N_{\omega}$-norm for each $t>0, x \in D\left(A^{\omega}\right)$. Since $D\left(A^{\omega}\right)$ is dense in $\mathscr{D}_{\omega}$, we may, using part (iii) of Proposition 2.3, extend these continuity properties to $\mathscr{D}_{\omega}$. Hence $\left\{T_{t}^{\omega}\right\}$ is of class $\left(C_{0}\right)$ on $\mathscr{D}_{\omega}$.

Now we introduce the (classical) infinitesimal generator of $\left\{T_{t}^{\omega}\right\}$, and call it $\tilde{A}^{\omega}$. Let

$$
N_{\omega}\left(T_{t}^{\omega}\right)=\sup _{N_{\omega}(x) \leqslant 1 ; x \in \Sigma_{\omega}} N_{\omega}\left(T_{t}^{\omega} x\right)
$$

and

$$
\omega_{0}=\lim _{t \rightarrow \infty} \frac{1}{t} \log N_{\omega}\left(T_{t}^{\omega}\right)
$$

be the type of $\left\{T_{t}^{\omega}\right\}$. By Proposition 2.3(iii), $N_{\omega}\left(T_{t}^{\omega}\right) \leqslant e^{\omega t}$, so that $\omega_{0} \leqslant \omega$. Hence if $x \in D\left(\tilde{A}^{\omega}\right)$, then for some $y \in \mathscr{D}_{\omega}$, and $\lambda \in C$ with $\operatorname{Re} \lambda>\omega$, we have (cf. [12, p. 344])

$$
x=\int_{0}^{\infty} e^{-\lambda t} T_{t} y d t
$$

and $\tilde{A}^{\omega} x=\lambda x-y$. But by $(2.20 .2)$,

$$
x \in \text { Range }\left(J_{\lambda}^{\omega} \mid \mathscr{D}_{\omega}\right)=\text { Range }\left(R\left(\lambda ; A^{\omega} \mid \mathscr{D}_{\omega}\right)\right)=\operatorname{Domain}\left(A^{\omega} \mid \mathscr{D}_{\omega}\right)
$$

(cf. Remark 2.19). Thus $x \in D\left(A^{\omega} \mid \mathscr{D}_{\omega}\right)$ and clearly $\left(A^{\omega} \mid \mathscr{D}_{\omega}\right) x=\lambda x-y$; so we have shown that $\tilde{A}^{\omega} \subset A^{\omega} \mid \mathscr{D}_{\omega}$.

We shall complete the proof by applying Lemma 2.17 . We have just seen that condition (i) is satisfied, and (ii) follows from the fact that 
$\{\lambda \in C: \operatorname{Re} \lambda>\omega\} \subset \rho\left(\tilde{A}^{\omega}\right) \cap \rho\left(A^{\omega} \mid \mathscr{D}_{\omega}\right)$,

since $\omega_{0} \leqslant \omega$ (cf. $(2.20 .1)$ ), and $\rho\left(A^{\omega}\right) \subset \rho\left(A^{\omega} \mid \mathscr{D}_{\omega}\right)$. Thus $\tilde{A}^{\omega}=A^{\omega} \mid \mathscr{D}_{\omega}$.

COROLlary 2.21. Let $\omega \in R$. Then the following hold:

(i) For all $x \in \mathscr{D}_{\omega}$,

$$
N_{\omega}\left(\lambda J_{\lambda}^{\omega} x-x\right) \rightarrow 0 \text { as } \lambda \rightarrow \infty
$$

(ii) For all integers $n \geqslant 1$,

$$
\overline{D\left(\left(A^{\omega}\right)^{n}\right)} N_{\omega}=\Phi_{\omega} ;
$$

(iii) For all integers $n \geqslant 1$,

$$
\overline{D\left(\left(A_{\omega}\right)^{n}\right)}=\bar{\Sigma}_{\omega} .
$$

Proof. (i) is clear, for any semigroup of class $\left(C_{0}\right)$ is necessarily of class $(A)$ (cf. [12, p. 321]). Of course, we refer to the semigroup $\left\{T_{t}^{\omega}\right\}_{t>0}$ on the Banach space $\left(\mathscr{D}_{\omega}, N_{\omega}\right)$. Or, for $x \in D\left(A^{\omega}\right)$, we have

$$
N_{\omega}\left(\lambda J_{\lambda}^{\omega} x-x\right)=N_{\omega}\left(J_{\lambda}^{\omega} A^{\omega} x\right) \leqslant N_{\omega}\left(A^{\omega} x\right) /(\lambda-\omega) \rightarrow 0 \text { as } \lambda \rightarrow \infty .
$$

(ii) In addition to (i), we have for $x \in D\left(A^{\omega}\right)$

$$
\begin{aligned}
N_{\omega}\left((\lambda-\omega) J_{\lambda}^{\omega} x-x\right) & =N_{\omega}\left(J_{\lambda}^{\omega} A^{\omega} x-\omega J_{\lambda}^{\omega} x\right) \leqslant N_{\omega}\left(A^{\omega} x-\omega x\right) /(\lambda-\omega) \\
& \rightarrow 0 \text { as } \lambda \rightarrow \infty .
\end{aligned}
$$

Thus for any $n \geqslant 1$,

$$
N_{\omega}\left((\lambda-\omega)^{n}\left(J_{\lambda}^{\omega}\right)^{n} x-x\right) \rightarrow 0 \text { as } \lambda \rightarrow \infty,
$$

because $N_{\omega}\left((\lambda-\omega)^{n}\left(J_{\lambda}^{\omega}\right)^{n}\right) \leqslant 1$ for $n \geqslant 1$. Since $\left(J_{\lambda}^{\omega}\right)^{n} x \in D\left(\left(A^{\omega}\right)^{n}\right)$, we see that $D\left(A^{\omega}\right) \subset \frac{{ }^{\omega}}{D\left(\left(A^{\omega}\right)^{n}\right)} N_{\omega}$, and (ii) now follows.

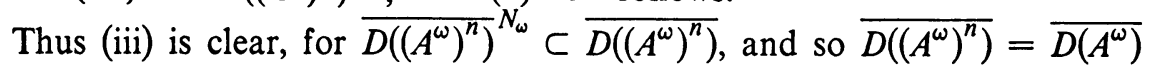
$=\bar{\Sigma}_{\omega}$, by Theorem 2.11(iv).

Theorem 2.20 leads to the following familiar representation of $\left\{T_{t}\right\}_{t>0}$.

COROLlaRy 2.22. Let $\omega \in R$, and suppose $x \in D\left(\left(A^{\omega}\right)^{2}\right)$. Then for each $t>0$,

$$
T_{t} x=\frac{1}{2 \pi i} \int_{\gamma-i \infty}^{\gamma+i \infty} e^{\lambda t} J_{\lambda}^{\omega} x d \lambda, \text { where } \gamma>\max (0, \omega) .
$$

(The integral converges in the $N_{\omega}$-norm, hence in the $X$-norm.) 
Proof. Let $x \in D\left(\left(A^{\omega}\right)^{2}\right)$. Then $x \in \mathscr{D}_{\omega}$, and $A^{\omega} x \in \mathscr{D}_{\omega}$, so

$$
x \in D\left(A^{\omega} \mid \mathscr{D}_{\omega}\right)=D\left(\tilde{A}^{\omega}\right),
$$

where $\tilde{A}^{\omega}$ denotes the classical infinitesimal generator of $\left\{T_{t}^{\omega}\right\}_{t>0}$. By Theorem 11.6.1 in [12], we have for $x \in D\left(\tilde{A}^{\omega}\right)$,

$$
T_{t} x=\lim _{\eta \rightarrow \infty} \frac{1}{2 \pi i} \int_{\gamma-i \eta}^{\gamma+i \eta} e^{\lambda t} R\left(\lambda ; \tilde{A}^{\omega}\right) x d \lambda, \quad \gamma>\max \left(0, \omega_{0}\right)
$$

where $\omega_{0}$ is the type of $\left\{T_{t}^{\omega}\right\}_{t>0}$ and the limit exists in the $N_{\omega}$-norm. But $R\left(\lambda ; \tilde{A}^{\omega}\right)=R\left(\lambda ; A^{\omega} \mid \mathscr{D}_{\omega}\right)=J_{\lambda}^{\omega} \mid \mathscr{D}_{\omega}$ for $\operatorname{Re} \lambda>\omega \geqslant \omega_{0}$. Thus (2.22.1) gives the desired result.

The integral representation of Corollary 2.22 is in fact valid for $x$ $\in D\left(A^{\omega} \mid \mathscr{D}_{\omega}\right)$, as may be observed in the proof. The next corollary also follows from the classical theory (cf. Theorem 11.6.6 in [12]).

Corollary 2.23. For $\omega \in R$ and $x \in \mathscr{D}_{\omega}$,

$$
T_{t} x=\lim _{k \rightarrow \infty}\left[\frac{k}{t} J_{k / t}^{\omega}\right]^{k} x=\lim _{k \rightarrow \infty}\left[I-\frac{t}{k}\left(A^{\omega} \mid \mathscr{D}_{\omega}\right)\right]^{-k} x,
$$

where the limits exist in the $N_{\omega}$-norm, hence in the $X$-norm.

In Theorem 2.27 we will show that the following necessary condition is one of a number of sufficient conditions that $A$ generate a semigroup of unbounded operators.

Corollary 2.24. Let $\omega \in R, t>0$. For $x \in D\left(\left(A^{\omega}\right)^{3}\right)$, define

$$
I_{\omega, t}(x)=\frac{1}{2 \pi i} \int_{0}^{t} \int_{\gamma-i \infty}^{\gamma+i \infty} e^{\lambda s} J_{\lambda}^{\omega} A^{\omega} x d \lambda d s, \quad \gamma>\max (0, \omega) .
$$

Then the operator $I_{\omega, t}$ is well defined, and is closable (in the $X$-norm).

Proof. Let $x \in D\left(\left(A^{\omega}\right)^{3}\right)$. Then $A^{\omega} x \in D\left(\left(A^{\omega}\right)^{2}\right)$, and by Corollary 2.22,

$$
T_{t} A^{\omega} x=\frac{1}{2 \pi i} \int_{\gamma-i \infty}^{\gamma+i \infty} e^{\lambda t} J_{\lambda}^{\omega} A^{\omega} x d \lambda, \quad \gamma>\max (0, \omega) .
$$

Since $A^{\omega} x \in \Sigma_{\omega}, T_{t} A^{\omega} x$ is a continuous function of $t$, for $t \geqslant 0$. Thus $I_{\omega, t}$ is well defined on $D\left(\left(A^{\omega}\right)^{3}\right)$.

Now let $\left\{x_{n}\right\} \subset D\left(\left(A^{\omega}\right)^{3}\right)$, and suppose $\left\|x_{n}\right\| \rightarrow 0$ and $\left\|I_{\omega, t}\left(x_{n}\right)-y\right\|$ $\rightarrow 0$ as $n \rightarrow \infty$ for some $y \in X$. By Theorem 2.13(ii) we have, for each $n$, 


$$
\begin{aligned}
T_{t} x_{n}-x_{n} & =\int_{0}^{t} A^{\omega} T_{s} x_{n} d s \\
& =\int_{0}^{t} A^{\omega}\left[\frac{1}{2 \pi i} \int_{\gamma-i \infty}^{\gamma+i \infty} e^{\lambda s} J_{\lambda}^{\omega} x_{n} d \lambda\right] d s, \text { for } \gamma>\max (0, \omega),
\end{aligned}
$$

where the inner integral converges (to $T_{s} x_{n}$ ) in the $N_{\omega}$-norm. Now $A^{\omega}$ is a closed operator in the $N_{\omega}$-norm topology, $J_{\lambda}^{\omega} x_{n} \in D\left(A^{\omega}\right)$, and

$$
\int_{\gamma-i \infty}^{\gamma+i \infty} e^{\lambda s} A^{\omega} J_{\lambda}^{\omega} x_{n} d \lambda=\int_{\gamma-i \infty}^{\gamma+i \infty} e^{\lambda s} J_{\lambda}^{\omega} A^{\omega} x_{n} d \lambda
$$

converges by Corollary 2.22 , since $A^{\omega} x_{n} \in D\left(\left(A^{\omega}\right)^{2}\right)$. Thus

$$
T_{t} x_{n}-x_{n}=\frac{1}{2 \pi i} \int_{0}^{t} \int_{\gamma-i \infty}^{\gamma+i \infty} e^{\lambda s} J_{\lambda}^{\omega} A^{\omega} x_{n} d \lambda d s=I_{\omega, t}\left(x_{n}\right) .
$$

(Observe that $I_{\omega, t}$ is independent of the choice of $\gamma$.) Since $T_{l} \mid \bar{\Sigma}_{\omega}$ is a closed operator in $\bar{\Sigma}_{\omega}$ (in the $X$-norm), the hypotheses imply that $y=0$. Thus $I_{\omega, t}$ is closable.

The next result characterizes the set $\mathscr{D}_{\omega}$ as the "continuity set" of the semigroup $\left\{T_{t} \mid \Sigma_{\omega}\right\}$ on the Banach space $\left(\Sigma_{\omega}, N_{\omega}\right)$ (cf. [8]); it also facilitates the proof of Theorem 2.26.

LEMMA 2.25. For each $\omega \in R$,

$$
\mathscr{D}_{\omega}={\overline{D\left(A^{\omega}\right)}}^{N_{\omega}}=\left\{x \in \Sigma_{\omega} \mid N_{\omega}\left(T_{t} x-x\right) \rightarrow 0 \text { as } t \rightarrow 0^{+}\right\} .
$$

Proof. Call the set on the right-hand side $\mathcal{C}_{\omega}$. It was shown in Theorem 2.20 that $\left\{T_{t} \mid \mathscr{D}_{\omega}\right\}_{t>0}$ is of class $\left(C_{0}\right)$ on $\left(\mathscr{D}_{\omega}, N_{\omega}\right)$, so $\mathscr{D}_{\omega} \subset \mathcal{C}_{\omega}$. On the other hand, if $x \in \mathcal{C}_{\omega}$, we shall show that $N_{\omega}\left(\lambda J_{\lambda}^{\omega} x-x\right) \rightarrow 0$ as $\lambda \rightarrow \infty$; the argument is similar to that in Theorem 2.8(ii).

Let $t>0$. Then

$$
e^{-\omega t}\left\|T_{t}\left(\lambda J_{\lambda}^{\omega} x-x\right)\right\| \leqslant e^{-\omega t} \lambda \int_{0}^{\infty} e^{-\lambda s}\left\|T_{t+s} x-T_{t} x\right\| d s .
$$

Given $\varepsilon>0$, let $\delta>0$ be such that $N_{\omega}\left(T_{s} x-x\right)<\varepsilon$ for $0<s<\delta$.

Splitting the ingegral in (2.25.1) from 0 to $\delta$, and from $\delta$ to $\infty$, we obtain

$$
\begin{aligned}
e^{-\omega t}\left\|T_{t}\left(\lambda J_{\lambda}^{\omega} x-x\right)\right\| & \leqslant \lambda \int_{0}^{\delta} e^{-\lambda s} N_{\omega}\left(T_{s} x-x\right) d s+\lambda \int_{\delta}^{\infty} e^{-\lambda s} N_{\omega}\left(T_{s} x-x\right) d s \\
& <\varepsilon\left(1-e^{-\lambda \delta}\right)+\lambda \int_{\delta}^{\infty} e^{-\lambda s}\left(e^{\omega s}+1\right) N_{\omega}(x) d s \\
& =\varepsilon\left(1-e^{-\lambda \delta}\right)+\frac{\lambda}{(\lambda-\omega)} e^{-(\lambda-\omega) \delta} N_{\omega}(x)+e^{-\lambda \delta} N_{\omega}(x) .
\end{aligned}
$$


Since this estimate of (2.25.1) is independent of $t$, we see that

$$
\varlimsup_{\lambda \rightarrow \infty} N_{\omega}\left(\lambda J_{\lambda}^{\omega} x-x\right) \leqslant \varepsilon .
$$

Thus $N_{\omega}\left(\lambda J_{\lambda}^{\omega} x-x\right) \rightarrow 0$ as $\lambda \rightarrow \infty$.

If $A$ is the infinitesimal generator of a semigroup of unbounded operators $\left\{T_{t}\right\}_{t>0}$ such that, for each $\omega \in R, T_{t} \mid \bar{\Sigma}_{\omega}$ is a closed operator, then we have various representations of the semigroup $\left\{T_{t}\right\}_{t>0}$ on suitable subspaces of $X$ (cf. Corollaries 2.22 and 2.23). In the next theorem, we prove that these representations are "maximal-unique" (cf. [14, Theorem 2.1]).

THEOREM 2.26. Let $A$ be the infinitesimal generator of a semigroup of unbounded linear operators $\left\{T_{t}\right\}_{t>0}$ such that, for each $\omega \in R, T_{t} \mid \bar{\Sigma}_{\omega}$ is a closed operator in $\bar{\Sigma}_{\omega}$. Then $A$ is "maximal-unique" in the following sense:

Suppose $\left\{\Sigma_{\omega}^{\prime}\right\}_{\omega \in R}$ is a nested family of subspaces on $X$, and $A^{\prime}$ a linear operator with domain and range in $\Sigma^{\prime}=\cup_{\omega \in R} \Sigma_{\omega}^{\prime}$ (and $A^{\omega^{\prime}}$ will denote $A^{\prime} \mid \Sigma_{\omega}^{\prime}$, the restriction of $A^{\prime}$ to $\Sigma_{\omega}^{\prime}$, with the usual domain) such that

(i) for every $x \in \Sigma_{\omega}^{\prime}$, the resolvent equation

$$
\left(\lambda I-A^{\prime}\right) y=x, \quad \operatorname{Re} \lambda>\omega,
$$

has a unique solution in $\Sigma_{\omega}^{\prime}$, say $J_{\lambda}^{\omega^{\prime}} x$;

(ii) $\Sigma_{\omega}^{\prime}$ is a Banach space with norm

$$
N_{\omega}^{\prime}(x)=\sup _{n \geqslant 0} \sup _{\lambda>\omega}\left\|(\lambda-\omega)^{n}\left(J_{\lambda}^{\omega^{\prime}}\right)^{n} x\right\|
$$

and

$$
N_{\omega}^{\prime}\left((\operatorname{Re} \lambda-\omega) J_{\lambda}^{\omega^{\prime}} x\right) \leqslant N_{\omega}^{\prime}(x) \text { for each } x \in \Sigma_{\omega}^{\prime} ;
$$

(iii) if $\mathscr{D}_{\omega}^{\prime}=\overline{\overline{D\left(A^{\omega^{\prime}}\right)}}{ }^{N_{\omega}^{\prime}}$, then $\mathscr{D}_{\omega}^{\prime} \subset \mathbf{D}(c f .(2.1 .1)), T_{t} \mathscr{D}_{\omega}^{\prime} \subset \mathscr{D}_{\omega}^{\prime}$ for each $t>0$, and $\left\{T_{t} \mid \Phi_{\omega}^{\prime}\right\}_{t>0}$ is a semigroup of bounded linear operators of class $\left(C_{0}\right)$ on the Banach space $\left(\mathscr{D}_{\omega}^{\prime}, N_{\omega}^{\prime}\right)$, with infinitesimal generator $A^{\omega^{\prime}} \mid \mathscr{D}_{\omega}^{\prime}$ (in the classical sense).

Then $\mathscr{D}_{\omega}^{\prime} \subset \mathscr{D}_{\omega}$, and $A^{\omega}\left|\mathscr{D}_{\omega}^{\prime}=A^{\omega^{\prime}}\right| \mathscr{D}_{\omega}^{\prime}$ (cf. Definition 2.18).

Proof. Fix $\omega \in R$. First, we show that, for each $x \in \mathscr{D}_{\omega}^{\prime}$,

$$
N_{\omega}^{\prime}(x)=\sup _{t>0} e^{-\omega t}\left\|T_{t} x\right\|=N_{\omega}(x) .
$$

Now $A^{\omega^{\prime}} \mid \mathscr{D}_{\omega}^{\prime}$ is the (classical) generator of $\left\{T_{t} \mid \mathscr{D}_{\omega}^{\prime}\right\}_{t>0}$, and $J_{\lambda}^{\omega^{\prime}}=R\left(\lambda ; A^{\omega^{\prime}}\right)$ by (i). Thus $J_{\lambda}^{\omega^{\prime}} \mid \mathscr{D}_{\omega}^{\prime}=R\left(\lambda ; A^{\omega^{\prime}} \mid \mathscr{D}_{\omega}^{\prime}\right)$,so for $x \in \mathscr{D}_{\omega}^{\prime}$ we have

$$
\left(J_{\lambda}^{\omega^{\prime}}\right)^{n} x=\frac{1}{(n-1) !} \int_{0}^{\infty} e^{-\lambda t} t^{n-1} T_{t} x d t, \quad n \geqslant 1 .
$$


Hence

$$
N_{\omega}^{\prime}(x) \leqslant \sup _{t>0} e^{-\omega t}\left\|T_{t} x\right\|
$$

On the other hand, since $N_{\omega}^{\prime}\left((\operatorname{Re} \lambda-\omega) J_{\lambda}^{\omega^{\prime}}\right) \leqslant 1$, we know from the classical theory that $N_{\omega}^{\prime}\left(T_{t} x\right) \leqslant e^{\omega t} N_{\omega}^{\prime}(x)$, for all $x \in \mathscr{D}_{\omega}^{\prime}$. Thus the reverse inequality holds, since (ii) implies $\left\|T_{t} x\right\| \leqslant N_{\omega}^{\prime}\left(T_{t} x\right)$ (let $n=0$ ).

This tells us that $\mathscr{D}_{\omega}^{\prime} \subset \Sigma_{\omega}$ (Definition 2.1). In fact, $\mathscr{D}_{\omega}^{\prime} \subset \mathscr{D}_{\omega}$; indeed, if $x \in \mathscr{D}_{\omega}^{\prime}$ we have by (iii) that $N_{\omega}^{\prime}\left(T_{t} x-x\right) \rightarrow 0$ as $t \rightarrow 0^{+}$. But $N_{\omega}^{\prime}\left(T_{t} x-x\right)$ $=N_{\omega}\left(T_{t} x-x\right)$, because $x \in \mathscr{D}_{\omega}^{\prime}$ implies $T_{t} x \in \mathscr{D}_{\omega}^{\prime}$, by (iii). Thus

$$
N_{\omega}\left(T_{t} x-x\right) \rightarrow 0 \text { as } t \rightarrow 0^{+},
$$

so, by Lemma $2.25, x \in \mathscr{D}_{\omega}$.

It now makes sense to speak of the restriction of $A^{\omega}$ to $\mathscr{D}_{\omega}^{\prime}$ (which we shall denote $A^{\omega} \mid \mathscr{D}_{\omega}^{\prime}$ ), with domain

$$
D\left(A^{\omega} \mid \mathscr{D}_{\omega}^{\prime}\right)=\left\{x \in \mathscr{D}_{\omega}^{\prime} \mid x \in D\left(A^{\omega}\right) \text { and } A^{\omega} x \in \mathscr{D}_{\omega}^{\prime}\right\} .
$$

It remains only to prove $A^{\omega}\left|\mathscr{D}_{\omega}^{\prime}=A^{\omega^{\prime}}\right| \mathscr{D}_{\omega}^{\prime}$.

Let $x \in D\left(A^{\omega} \mid \mathscr{D}_{\omega}^{\prime}\right)$. Then for some $y \in \mathscr{D}_{\omega}^{\prime}$ and $\operatorname{Re} \lambda>\omega$,

$$
x=\int_{0}^{\infty} e^{-\lambda t} T_{t} y d t
$$

and $A^{\omega} x=\lambda x-y$. But $\operatorname{Re} \lambda>\omega$ implies $\lambda \in \rho\left(A^{\omega^{\prime}} \mid \mathscr{D}_{\omega}^{\prime}\right)$, since the type of $\left\{T_{t} \mid \mathscr{D}_{\omega}^{\prime}\right\}_{t>0}$ is $\leqslant \omega$ (for $\left.N_{i}^{\prime}\left(T_{t} \mid \mathscr{D}_{\omega}^{\prime}\right) \leqslant e^{\omega t}\right)$. Thus the integral (2.26.1) exists in the $N_{\omega^{\prime}}^{\prime}$-norm, and represents the resolvent of $A^{\omega^{\prime}} \mid \mathscr{D}_{\omega}^{\prime}$. Therefore $x$ $\in D\left(A^{\omega^{\prime}} \mid \mathscr{D}_{\omega}^{\prime}\right)$, and $A^{\omega^{\prime}} x=\lambda x-y$, so $A^{\omega}\left|\mathscr{D}_{\omega}^{\prime} \subset A^{\omega^{\prime}}\right| \Phi_{\omega}^{\prime}$.

Conversely, let $x \in D\left(A^{\omega^{\prime}} \mid \mathscr{D}_{\omega}^{\prime}\right)$. Then (2.26.1) holds for some $y \in \mathscr{D}_{\omega}^{\prime}$ $\subset \mathscr{D}_{\omega}$, and $A^{\omega^{\prime}} x=\lambda x-y \in \mathscr{D}_{\omega}^{\prime}$. Thus $x \in D\left(A^{\omega}\right)$, and $A^{\omega} x=\lambda x-y$ $=A^{\omega^{\prime}} x \in \mathscr{D}_{\omega}^{\prime}$. Therefore $x \in D\left(A^{\omega} \mid \mathscr{D}_{\omega}^{\prime}\right)$, and so $A^{\omega}\left|\mathscr{D}_{\omega}^{\prime}=A^{\omega^{\prime}}\right| \mathscr{D}_{\omega}^{\prime}$.

In view of the preceding theorem, we shall call any operator satisfying the hypotheses of Theorem 2.26 an infinitesimal operator of $\left\{T_{t}\right\}_{t>0}$.

We now prove a converse theorem which characterizes those operators $A$ which serve as infinitesimal generators of certain semigroups of unbounded linear operators.

THEOREM 2.27. Let $X$ be a Banach space, and let $\left\{\Sigma_{\omega}\right\}_{\omega \in R}$ be a nested family of nontrivial subspaces of $X$, and $A$ a linear operator with domain and range in $\Sigma=\cup_{\omega \in R} \Sigma_{\omega}$ (and $A^{\omega}$ will denote $A \mid \Sigma_{\omega}$, the restriction of $A$ to $\Sigma_{\omega}$, with the usual domain). Assume that for each $x \in \Sigma_{\omega}$, the resolvent equation

$$
(\lambda I-A) y=x, \quad \operatorname{Re} \lambda>\omega,
$$


has a unique solution, say $J_{\lambda}^{\omega} x$, belonging to $\Sigma_{\omega^{*}}$ (Note that $J_{\lambda}^{\omega}: \Sigma_{\omega} \rightarrow \Sigma_{\omega}$ is a linear operator on $\Sigma_{\omega}$ )

Assume also that if $N_{\omega}(x)=\sup _{n \geq 0} \sup _{\lambda>\omega}\left\|(\lambda-\omega)^{n}\left(J_{\lambda}^{\omega}\right)^{n} x\right\|$, then $N_{\omega}(x)$ is finite for each $x \in \Sigma_{\omega}$, and $\left(\Sigma_{\omega}, N_{\omega}\right)$ is a Banach space; in addition, suppose the following hold:

(i) $N_{\omega}\left((\operatorname{Re} \lambda-\omega) J_{\lambda}^{\omega} x\right) \leqslant N_{\omega}(x)$, for $\omega \in R, \operatorname{Re} \lambda>\omega, x \in \Sigma_{\omega}$;

(ii) for all $x \in \Sigma_{\omega}$, $\left\|\lambda J_{\lambda}^{\omega} x-x\right\| \rightarrow 0$ as $\lambda \rightarrow \infty$;

(iii) for each $\omega \in R, t>0$, the operator

$$
I_{\omega, t}(x)=\frac{1}{2 \pi i} \int_{0}^{t} \int_{\gamma-i \infty}^{\gamma+i \infty} e^{\lambda s} J_{\lambda}^{\omega} A^{\omega} x d \lambda d s, \quad \text { where } \gamma<\max (0, \omega),
$$

defined on $D\left(\left(A^{\omega}\right)^{3}\right)$, is closable (in the $X$-norm).

Then there exists a one-parameter family $\left\{T_{t}\right\}_{t>0}$ of (in general unbounded) linear operators in $X$ such that

(i') if $\mathscr{D}_{\omega}=\overline{D\left(A^{\omega}\right)}{ }^{N_{\omega}}$, then $\mathscr{D}=\cup_{\omega \in R} \mathscr{D}_{\omega} \subset \mathbf{D}(c f .(2.1 .1))$;

(ii') for each $\omega \in R, T_{t} \mid \bar{\Sigma}_{\omega}$ is a closed operator in $\bar{\Sigma}_{\omega}$, for every $t>0$;

(iii') if $\tilde{A}$ is the infinitesimal generator of $\left\{T_{t}\right\}_{t>0}, \tilde{N}_{\omega}$ and $\tilde{\Sigma}_{\omega}$ are as in Definition 2.1 , and $\tilde{A}^{\omega}=\tilde{A} \mid \tilde{\Sigma}_{\omega}$, then for each $\left.\omega \in R, \mathscr{D}_{\omega} \subset \tilde{D}^{\overline{A^{\omega}}}\right)^{N_{\omega}}$ and $A^{\omega} \mid \mathscr{D}_{\omega}$ $=\left.\tilde{A}^{\omega}\right|_{\omega}$.

Proof. We make a number of preliminary observations:

(1) For each $\omega \in R, J_{\lambda}^{\omega}$ is the resolvent of $A^{\omega}$, for $\operatorname{Re} \lambda>\omega$. By (i), $J_{\lambda}^{\omega}$ is bounded on the Banach space $\left(\Sigma_{\omega}, N_{\omega}\right)$, so $A^{\omega}$ is a closed operator in the $N_{\omega}$ norm topology.

(2) For each $\omega \in R$ and $x \in \Sigma_{\omega},\|x\| \leqslant N_{\omega}(x)$.

(3) Since $J_{\lambda}^{\omega} x \in D\left(A^{\omega}\right)$ for each $x \in \Sigma_{\omega}$, (ii) implies that $\overline{D\left(A^{\omega}\right)}=\bar{\Sigma}_{\omega}$. Thus $\overline{D(A)}=\overline{\cup_{\omega \in R} D\left(A^{\omega}\right)}=\bar{\Sigma}$.

(4) If $\omega_{1} \leqslant \omega_{2}, x \in \Sigma_{\omega_{1}} \subset \Sigma_{\omega_{2}}$, and $\operatorname{Re} \lambda>\omega_{2}$, then $J_{\lambda}^{\omega_{1}} x=J_{\lambda}^{\omega_{2}} x$. Indeed, both are solutions of the resolvent equation $(\lambda I-A) y=x$ belonging to $\Sigma_{\omega_{2}}$. Thus $J_{\lambda}^{\omega_{1}} \subset J_{\lambda}^{\omega_{2}}$ whenever $\operatorname{Re} \lambda>\omega_{2}$, and so $A^{\omega_{1}} \subset A^{\omega_{2}}$.

(5) If $\omega_{1} \leqslant \omega_{2}$, and $x \in \Sigma_{\omega_{1}}$, then $N_{\omega_{2}}(x) \leqslant N_{\omega_{1}}(x)$, since $J_{\lambda}^{\omega_{2}} x$ $=J_{\lambda}^{\omega_{1}} x$ for $\lambda>\omega_{2}$, and $\left\|\left(\lambda-\omega_{2}\right)^{n}\left(J_{\lambda}^{\omega_{1}}\right)^{n} x\right\| \stackrel{\omega_{2}}{ }\left\|\left(\lambda-\omega_{1}\right)^{n}\left(J_{\lambda}^{\omega_{1}}\right)^{n} x\right\|$.

(6) $I_{\omega, t}$ is a well-defined operator on $D\left(\left(A^{\omega}\right)^{3}\right)$ (cf. Lemma 11.5.2 in [12]).

Now fix $\omega \in R$, and consider the Banach space $\mathscr{D}_{\omega}=\overline{D\left(A^{\omega}\right)}{ }^{N_{\omega}}$, with norm $N_{\omega}$. Let $A^{\omega} \mid \mathscr{D}_{\omega}$ be the restriction of $A^{\omega}$ to $\mathscr{D}_{\omega}$. Then, by Remark 2.19, $A^{\omega} \mid \mathscr{D}_{\omega}$ is a closed, densely-defined operator in $\mathscr{D}_{\omega}$ with resolvent $J_{\lambda}^{\omega} \mid \mathscr{D}_{\omega}$ for $\operatorname{Re} \lambda>\omega$. By (i), $N_{\omega}\left((\operatorname{Re} \lambda-\omega) J_{\lambda}^{\omega} \mid \mathscr{D}_{\omega}\right) \leqslant 1$, so we are in a position to invoke the classical Hille-Yosida theorem (cf. Theorem 12.3 .1 in [12]). Thus $A^{\omega} \mid \mathscr{D}_{\omega}$ is the (classical) infinitesimal generator of a semigroup of bounded linear operators of class $\left(C_{0}\right)$ on $\mathscr{D}_{\omega}$, which we shall denote $\left\{T_{t}^{\omega}\right\}_{t>0}$. In addition, we obtain from the classical theory that $N_{\omega}\left(T_{t}^{\omega}\right) \leqslant e^{\omega t}$. 
Repeating the above procedure for each $\omega \in R$, we then define, for $x \in \cup_{\omega \in R} \mathscr{D}_{\omega}, t>0, T_{t} x=T_{t}^{\omega} x$, if $x \in \mathscr{D}_{\omega} . T_{t}$ is well defined for each $t>0$, because if $\omega_{1} \leqslant \omega_{2}$, then $\mathscr{D}_{\omega_{1}} \subseteq \mathscr{D}_{\omega_{2}}$. Indeed, by (4), $D\left(A^{\omega_{1}}\right) \subset D\left(A^{\omega_{2}}\right)$,

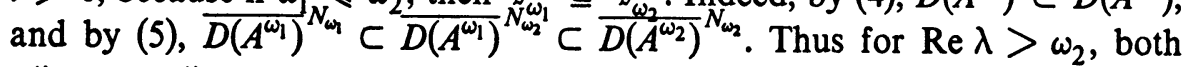
$J_{\lambda}^{\omega_{1}} x$ and $J_{\lambda}^{\omega_{2}} x$ are defined, and equal by (4). From the classical theory,

$$
J_{\lambda}^{\omega_{1}} x=\int_{0}^{\infty} e^{-\lambda t} T_{t}^{\omega_{1}} x d t=J_{\lambda}^{\omega_{2}} x=\int_{0}^{\infty} e^{-\lambda t} T_{t}^{\omega_{2}} x d t .
$$

By uniqueness of the Laplace transform, it now follows that $T_{t}^{\omega_{1}} x=T_{t}^{\omega_{2}} x$, so $T_{t}$ is well defined (in fact, $T_{t}^{\omega_{1}} \subset T_{t}^{\omega_{2}}$ ).

At this point, it is clear that the operators $\left\{T_{t}\right\}_{t>0}$ satisfy the semigroup property on $\mathscr{D}=\cup_{\omega \in R} \mathscr{D}_{\omega}$, that $T_{t} x$ is strongly continuous for $t>0$, and $\left\|T_{t} x-x\right\| \rightarrow 0$ as $t \rightarrow 0^{+}$. (In fact, the continuity properties hold in the $N_{\omega}$-norm, for appropriate $\omega \in R$.)

Now we know from the classical theory that, for $x \in D\left(A^{\omega} \mid \mathscr{D}_{\omega}\right)$,

$$
T_{t}^{\omega} x-x=\int_{0}^{t} A^{\omega} T_{s}^{\omega} x d s
$$

and for $s>0$,

$$
T_{s}^{\omega} x=\frac{1}{2 \pi i} \int_{\gamma-i \infty}^{\gamma+i \infty} e^{\lambda s} J_{\lambda}^{\omega} x d \lambda, \quad \gamma>\max (0, \omega) .
$$

In particular, (2.27.2) and (2.27.3) hold for $x \in D\left(\left(A^{\omega}\right)^{3}\right)$. By substituting (2.27.3) into (2.27.2), and using remark (1) above (also cf. the proof of Corollary 2.24), we see that, for $x \in D\left(\left(A^{\omega}\right)^{3}\right), I_{\omega, t}(x)=T_{t}^{\omega} x-x$. Thus $T_{t}^{\omega}$, with domain $D\left(\left(A^{\omega}\right)^{3}\right)$, is a closable operator, by (iii). Denote the closure by $\overline{T_{t}^{\omega}}$, and define $\tilde{T}_{t} x=\bar{T}_{t}^{\omega} x$, if $x \in D\left(\overline{T_{t}^{\omega}}\right) . \tilde{T}_{t}$ is well defined, for if $\omega_{1} \leqslant \omega_{2}$, then $\overline{T_{t}^{\omega_{1}}} \subset \overline{T_{t}^{\omega_{2}}}$.

Since $T_{t}$ has already been defined on the whole of $\mathscr{D}=\cup_{\omega \in R} \mathscr{D}_{\omega}$, we must check that our extended operators $\tilde{T}_{t}$ include $\mathscr{D}$ in their domain, and that $\tilde{T}_{t}$ agrees with $T_{t}$ on $\mathscr{D}$. We show that, for each $\omega \in R, \Phi_{\omega} \subset D\left(\overline{T_{t}^{\omega}}\right)$, and $T_{t}^{\omega}=\overline{T_{t}^{\omega}}$ on $\mathscr{D}_{\omega}$. So let $x \in \mathscr{D}_{\omega}$. Then there exists a sequence $\left\{x_{n}\right\} \subset D\left(\left(A^{\omega}\right)^{3}\right)$ such that $N_{\omega}\left(x-x_{n}\right) \rightarrow 0$ as $n \rightarrow \infty$, since the proof of Corollary 2.21(ii) shows that for $n \geqslant 1, \overline{D\left(\left(A^{\omega}\right)^{n}\right)^{n}}=\mathscr{D}_{\omega}$. But $T_{t}^{\omega}$ is a bounded operator on $\left(D_{\omega}, N_{\omega}\right)$, so $N_{\omega}\left(T_{t} x-T_{t} x_{n}\right) \rightarrow 0$ as $n \rightarrow \infty$. By (2), the $N_{\omega}$-norm is stronger than the norm of $X$, so that $\left\|x-x_{n}\right\| \rightarrow 0$ and $\left\|T_{t}^{\omega} x-T_{t}^{\omega} x_{n}\right\| \rightarrow 0$ as $n \rightarrow \infty$. Thus $x \in D\left(\overline{T_{t}^{\omega}}\right)$, and $\overline{T_{t}^{\omega}} x=T_{t}^{\omega} x$.

We now rename $\tilde{T}_{t}$ by $T_{t}$; thus we have a one-parameter family $\left\{T_{t}\right\}_{t>0}$ of linear operators on $X$ such that $\mathscr{D}=\cup_{\omega \in R} \mathscr{D}_{\omega} \subset \mathbf{D}$, so (i') is proved.

For (ii'), observe first that for each $\omega \in R, D\left(\left(A^{\omega}\right)^{3}\right)=\bar{\Sigma}_{\omega}$. Indeed, in (3) we remarked that $\overline{D\left(A^{\omega}\right)}=\bar{\Sigma}_{\omega}$, and $\overline{D\left(\left(A^{\omega}\right)^{n}\right)}=\overline{D\left(A^{\omega}\right)}$ for $n \geqslant 1$ is proved 
exactly as in Corollary 2.21(iii). Now we have, by construction, that

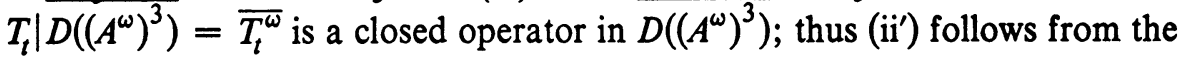
preceding observation.

Next we introduce the spaces $\left(\tilde{\boldsymbol{\Sigma}}_{\omega}, \tilde{N}_{\omega}\right)$, as defined in (2.1). Since we have proved (ii'), these are Banach spaces; let the infinitesimal generator, $\tilde{A}$, be as in Definition 2.10. It is clear that the operator $A$, and the spaces $\left\{\Sigma_{\omega}\right\}_{\omega \in R}$, satisfy the requirements of Theorem 2.26 . Thus by "maximal-uniqueness" we have, for each $\omega \in R$,

$$
\mathscr{D}_{\omega} \subset \overline{D\left(\tilde{A}^{\omega}\right)} \tilde{N}_{\omega} \text {, and } A^{\omega}\left|\mathscr{D}_{\omega}=\tilde{A}^{\omega}\right| \mathscr{D}_{\omega} .
$$

Remark. The semigroup $\left\{T_{t}\right\}_{t}>0$ obtained in Theorem 2.23 is uniquely determined by the operator $A$ on the set $\mathscr{D}=\cup_{\omega \in R} \mathscr{D}_{\omega}$. In other words, $A$ is an "infinitesimal operator" of $\left\{T_{t}\right\}_{t}>0$; the operators $T_{t}$ may have extensions for which $A$ is also an infinitesimal operator. We wish also to emphasize that although the domain of $A$ was assumed to be nontrivial, this is not necessarily the case (cf. Remark 2.12).

3. Illustrations. We now consider several concrete examples which motivate our theory and demonstrate the variety of possibilities for the domain of the infinitesimal generator of a semigroup of unbounded operators. Throughout this section, the sets $\mathbf{D}, \Sigma_{\omega}$ and $\Sigma$ will be defined as in $\$ 2$.

Fractional integrals in $L^{p}(0, \infty), 1<p<\infty$, provide a model for the theory developed in $\$ 2$. We first consider several properties of the RiemannLiouville fractional integral as an unbounded operator in $L^{p}(0, \infty)$.

Let $f$ be a real or complex-valued Lebesgue measurable function defined on $(0, \infty)$, such that for every $N>0, f \in L^{1}(0, N)$. For $\alpha \in C$ with $\operatorname{Re} \alpha>0$, define the Riemann-Liouville fractional integral

$$
I^{\alpha} f(x)=\frac{1}{\Gamma(\alpha)} \int_{0}^{x}(x-t)^{\alpha-1} f(t) d t .
$$

$I^{\alpha} f$ exists for almost all $x$ in $(0, \infty)$, and also belongs to $L^{1}(0, N)$ for each $N>0$. If we let $I^{\alpha}$ act on $L^{p}(0, N), 1 \leqslant p<\infty$, then $I^{\alpha}$ is a bounded linear operator; in fact, $\left\{I^{\alpha}\right\}_{\operatorname{Re} \alpha>0}$ forms a holomorphic semigroup on $L^{p}(0, N)$ (cf. $\$ 23.6$ in [12]). Also, for $f \in L^{p}(0, N), 1<p<\infty$, and $\gamma \in R$,

$$
\lim _{\varepsilon \rightarrow 0^{+}} I^{\varepsilon+i \gamma} f=I^{i \gamma} f
$$

exists in $L^{p}(0, N)$, and $\left\{I^{i \gamma}\right\}_{\gamma \in R}$ forms a strongly continuous group on $L^{p}(0, N)$. This result was proved for the case $p=2$ by Kober [17], and independently by Fisher [9] and Kalisch [13] for $1<p<\infty$.

We shall consider $I^{\alpha}$ as an operator in $L^{p}(0, \infty), 1<p<\infty$, with domain 


$$
D\left(I^{\alpha}\right)=\left\{f \in L^{p}(0, \infty) \mid I^{\alpha} f \in L^{p}(0, \infty)\right\},
$$

in which case it is unbounded.

Throughout this section, $I^{\alpha}$ will have domain as in (3.1.1), unless otherwise specified.

Proposition 3.1. Let $\alpha \in C, \operatorname{Re} \alpha>0$. Then $I^{\alpha}$ is a closed operator in $L^{p}(0, \infty), 1<p<\infty$.

Proof. Let $\left\{f_{n}\right\} \subset D\left(I^{\alpha}\right)$, and suppose there exist $f, g \in L^{p}(0, \infty)$ such that $f_{n} \stackrel{L^{p}}{\longrightarrow} f$ and $I^{\alpha} f_{n} \stackrel{L^{p}}{\longrightarrow} g$. Let $\hat{f}(\lambda)$ denote the Laplace transform of $f$. Then $\bar{I}^{\alpha} f(\lambda)$ converges absolutely for $\lambda \in C$ with $\operatorname{Re} \lambda>0$, and $\widehat{I^{\alpha} f}(\lambda)=\lambda^{-\alpha} \hat{f}(\lambda)$, $\operatorname{Re} \lambda>0$ (cf. [23, Chapter II, §8]).

Then for each $\lambda$ with $\operatorname{Re} \lambda>0,\left|\widehat{f}_{n}(\lambda)-\hat{f}(\lambda)\right| \rightarrow 0$, and $\left|\widehat{I^{\alpha} f_{n}}(\lambda)-\hat{g}(\lambda)\right|$ $=\left|\lambda^{-\alpha} \widehat{f_{n}}(\lambda)-\hat{g}(\lambda)\right| \rightarrow 0$ as $n \rightarrow \infty$. Thus $\left|\lambda^{-\alpha} \widehat{f_{n}}(\lambda)-\lambda^{-\alpha} \hat{f}(\lambda)\right| \rightarrow 0$, and so $\lambda^{-\alpha} \hat{f}(\lambda)=\hat{g}(\lambda)$. But $I^{\alpha} f$, which exists a.e. in $(0, \infty)$, has Laplace transform $\lambda^{-\alpha} \hat{f}(\lambda)$. By uniqueness of the Laplace transform, it now follows that $I^{\alpha} f=g$ a.e., so $f \in D\left(I^{\alpha}\right)$, and $I^{\alpha}$ is closed.

Although the next theorem is known (cf. Berens and Westphal [4, Proposition 6]), its statement as presented here is different from that in [4], where derivatives of fractional order are considered. (For related results, cf. Hardy, Landau and Littlewood [10], Kober [16], and Tamarkin [22].)

We shall need the following:

Lemma 3.2. For $\alpha>0, \gamma \in R, D\left(I^{\alpha}\right)=D\left(I^{\alpha+i \gamma}\right)$.

Proof. Following the notation used in Fisher [9], let

$$
T_{\varepsilon}^{\gamma} f(x)=\frac{1}{\Gamma(i \gamma)}\left[\int_{\varepsilon}^{\infty} f(x-y) y^{i \gamma-1} d y+\frac{1}{i \gamma} \varepsilon^{i \gamma} f(x)\right],
$$

where $f \in L^{p}(0, \infty), \gamma \in R$ and $\varepsilon>0$. Then $T^{\gamma} f=\lim _{\varepsilon \rightarrow 0^{+}} T_{\varepsilon}^{\gamma} f$ exists in $L^{p}(0, \infty)$ (and pointwise a.e.); moreover, $\left\{T^{\gamma}\right\}_{\gamma \in R}$ is a strongly continuous group of bounded operators on $L^{p}(0, \infty)$.

By Corollary 2.2 in [9], we have $T^{\gamma} I^{\alpha}=I^{\alpha} T^{\gamma}=I^{\alpha+i \gamma}$ as operators in $L^{p}(0, \infty)$. Since $T^{\gamma}$ is invertible, the result follows immediately.

THEOREM 3.3. As operators in $L^{p}(0, \infty), 1<p<\infty$,

$$
I^{\alpha} I^{\beta}=I^{\alpha+\beta}, \quad \operatorname{Re} \alpha, \operatorname{Re} \beta>0 .
$$

Proof. That $I^{\alpha} I^{\beta} \subseteq I^{\alpha+\beta}$ follows at once from Fubini's theorem. The other direction is equivalent to:

(3.3.1) If $f \in L^{p}(0, \infty)$, and $I^{\alpha} f \in L^{p}(0, \infty)$, where $\operatorname{Re} \alpha>0$, then $I^{\beta} f$ $\in L^{p}(0, \infty)$ whenever $0<\operatorname{Re} \beta<\operatorname{Re} \alpha$. 
For $\alpha, \beta$ real, (3.3.1) is equivalent to the statement of Proposition 6 in [4]. Applying Lemma 3.2, we obtain the desired result.

The following is now clear:

Corollary 3.4. (i) Whenever $\alpha, \beta \in C$ with $\operatorname{Re} \alpha>\operatorname{Re} \beta>0$, then $D\left(I^{\alpha}\right)$ $\subseteq D\left(I^{\beta}\right)$.

(ii) $\bigcap_{\alpha \in C ; \operatorname{Re} \alpha>0} D\left(I^{\alpha}\right)=\bigcap_{n \in Z^{+}} D\left(I^{n}\right)$.

Next we show that the operators $I^{\alpha}$ are densely defined in $L^{p}(0, \infty)$. In order to do this, we introduce the classical Weyl fractional integral: for $f$ $\in L^{p}(0, \infty)$, define

$$
J^{\alpha} f(x)=\frac{1}{\Gamma(\alpha)} \int_{x}^{\infty}(t-x)^{\alpha-1} f(t) d t, \quad \alpha \in C, \operatorname{Re} \alpha>0 .
$$

Suppose we denote by $\dot{J}^{\alpha}$ the operator $J^{\alpha}$ with domain $C_{c}(0, \infty)$, the set of continuous functions on $(0, \infty)$ with compact support. Then certainly $j^{\alpha}$ is a densely defined operator in $L^{p}(0, \infty)$; moreover, if $n \in Z^{+}$, then $j^{n}$ is closable. Indeed, if $J^{(1)}$ denotes the operator $J^{1}$ with maximal domain

$$
D\left(J^{(1)}\right)=\left\{f \in L^{p}(0, \infty) \mid J^{1} f \in L^{p}(0, \infty)\right\},
$$

then $J^{(n)}$, the operator $J^{(1)}$ applied $n$ times, is the inverse of the closed operator $D^{(n)}=(-1)^{n} f^{(n)}$, the $n$th derivative of $f$, with domain

$$
\operatorname{Domain}\left(D^{(n)}\right)=\left\{\begin{array}{l|l}
f \in L^{p}(0, \infty) & \begin{array}{l}
f^{(k)} \in L^{p}(0, \infty), 1 \leqslant k \leqslant n ; \\
f^{(k)} \text { absolutely continuous } \\
\text { on }(0, \infty), 1 \leqslant k \leqslant n-1
\end{array}
\end{array}\right\} .
$$

Thus $J^{(n)}$ is closed, and since $\dot{J}^{n} \subset J^{(n)}$, it follows that $\dot{J}^{n}$ is closable.

Since the operators $\dot{J}^{n}$ are densely defined, the adjoint operators $\left(\dot{J}^{n}\right)^{*}$ are well defined in $L^{q}(0, \infty), q=p /(p-1)$, where $\left(j^{n}\right)^{*}$ has domain

$$
D\left(\left(\dot{J}^{n}\right)^{*}\right)=\left\{\begin{array}{l|l}
f \in L^{p}(0, \infty) & \begin{array}{l}
\exists h \in L^{q}(0, \infty) \text { such that for } \\
\text { each } g \in D\left(\dot{J}^{n}\right),\left(f, \dot{J}^{n} g\right)=(h, g)
\end{array}
\end{array}\right\},
$$

and $\left(\dot{J}^{n}\right)^{*} f=h$ if $f \in D\left(\left(\dot{J}^{n}\right)^{*}\right)$ (cf. Chapter III, $\$ 5$ in [15]). Moreover, $\left(\dot{J}^{n}\right)^{*}$ is densely defined in $L^{q}(0, \infty)$, since $L^{p}(0, \infty)$ is reflexive, and $j^{n}$ is closable and densely defined (cf. [15, Theorem 5.29]).

With these remarks in mind, we now prove

Proposition 3.5. For each $\alpha \in C$ with $\operatorname{Re} \alpha>0, D\left(I^{\alpha}\right)$ is dense in $L^{p}(0, \infty), 1<p<\infty$.

PRoof. By Corollary 3.4(i), it suffices to prove the result for $\alpha=n \in Z^{+}$; we shall accomplish the proof by showing that for each $n \in Z^{+}, I^{n}=\left(\dot{J}^{n}\right)^{*}$, 
where $\dot{J}^{n}$ is now regarded as an operator in $L^{q}(0, \infty), q=p /(p-1)$.

Let $f \in D\left(I^{n}\right)$. Then if $g \in D\left(\dot{J}^{n}\right)$,

$$
\begin{aligned}
\int_{0}^{\infty} f(x) \dot{J}^{n} g(x) d x & =\int_{0}^{\infty} f(x) \frac{1}{\Gamma(n)} \int_{x}^{\infty}(t-x)^{n-1} g(t) d t d x \\
& =\int_{0}^{\infty} g(t) \frac{1}{\Gamma(n)} \int_{0}^{t}(t-x)^{n-1} f(x) d x d t \\
& =\int_{0}^{\infty} g(t) I^{n} f(t) d t
\end{aligned}
$$

the interchange of the order of integration is justified since $J^{n}|g| \in L^{q}(0, \infty)$, so the iterated integral in (3.5.1) converges absolutely. $I^{n} f \in L^{p}(0, \infty)$, so we have $f \in D\left(\left(\dot{J}^{n}\right)^{*}\right)$, and $\left(\dot{J}^{n}\right)^{*} f=I^{n} f$.

On the other hand, suppose $f \in D\left(\left(\dot{J}^{n}\right)^{*}\right)$. Then for all $g \in D\left(\dot{J}^{n}\right)$, there exists $h \in L^{p}(0, \infty)$ such that

$$
\int_{0}^{\infty} f(x) \dot{J}^{n} g(x) d x=\int_{0}^{\infty} h(x) g(x) d x .
$$

But since $\dot{J}^{n}|g| \in L^{q}(0, \infty)$, we may interchange the order of integration on the left side of (3.5.2) and obtain

$$
\int_{0}^{\infty} I^{n} f(x) g(x) d x=\int_{0}^{\infty} h(x) g(x) d x,
$$

where $I^{n} f(x)$ is a locally integrable function defined a.e. in $(0, \infty)$. Since (3.5.3) holds for all $g \in C_{c}(0, \infty), I^{n} f=h$ a.e. on every compact subset of $(0, \infty)$; hence $I^{n} f=h=\left(\dot{J}^{n}\right)^{*} f$ a.e. on $(0, \infty)$, and so $I^{n} f \in L^{p}(0, \infty)$. Therefore $I^{n}=\left(\dot{J}^{n}\right)^{*}$, so $I^{n}$ is densely defined.

REMARK. Let $\psi_{k}(x)=\left(d^{k} / d x^{k}\right)\left(x^{k} e^{-x}\right), k \in Z^{+}$. Then it is easy to see that $\psi_{k} \in D\left(I^{n}\right)$ if and only if $k \geqslant n$.

For applications of our theory, the Weyl fractional integral is somewhat more tenable. In the following development, we see its close relationship to the semigroup $\left\{I^{\alpha}\right\}_{\alpha>0}$.

Since each $I^{\alpha}$ is densely defined in $L^{p}(0, \infty), 1<p<\infty$ (Proposition 3.5), the adjoint operators $\left(I^{\alpha}\right)^{*}$ are well defined in $L^{q}(0, \infty)$, where $q=p /(p-1)$; for each $\alpha \in C$ with $\operatorname{Re} \alpha>0,\left(I^{\alpha}\right)^{*}$ is a closed linear operator with domain

$$
D\left(\left(I^{\alpha}\right)^{*}\right)=\left\{\begin{array}{l|l}
f \in L^{q}(0, \infty) & \begin{array}{l}
\exists h \in L^{q}(0, \infty) \text { such that, for } \\
\text { each } g \in D\left(I^{\alpha}\right),\left(f, I^{\alpha} g\right)=(h, g)
\end{array}
\end{array}\right\},
$$

and $\left(I^{\alpha}\right)^{*} f=h$ for $f \in D\left(\left(I^{\alpha}\right)^{*}\right)$.

Now consider the one-parameter family ofoperators $\left\{\left(I^{\alpha}\right)^{*}\right\}_{\alpha>0}$ (our theory applies only to real values of the parameter); we shall prove that $\left\{\left(I^{\alpha}\right)^{*}\right\}_{\alpha>0}$ is 
a semigroup of closed linear operators in $L^{q}(0, \infty), 1<q<\infty$, with densely defined infinitesimal generator. We accomplish this in a series of lemmas, and shall need the following known results.

In [16] and [17], Kober studies (among others) the operators

$$
K_{\eta, \alpha}^{-} f(x)=\frac{1}{\Gamma(\alpha)} x^{\eta} \int_{x}^{\infty}(t-x)^{\alpha-1} t^{-\eta-\alpha} f(t) d t,
$$

where $f \in L^{p}(0, \infty), 1 \leqslant p \leqslant \infty$, and $\alpha, \eta \in C$ with $\operatorname{Re} \alpha>0$. Then if $\operatorname{Re} \eta$ $>-1 / p, K_{\eta, \alpha}^{-}$exists a.e. in $(0, \infty)$, and

$$
\left\|K_{\eta, \alpha}^{-} f\right\|_{L^{p}(0, \infty)} \leqslant M\|f\|_{L^{p}(0, \infty)},
$$

where $M=M(p, \alpha, \eta)([16$, Therorem 2]). Kober's theorem is actually more general than this, but we need only (3.5.6). In fact, for the case $\eta=0$, (3.5.6) was proved by Hardy and Littlewood [11].

Moreover, if $f \in L^{p}(0, \infty), 1 \leqslant p<\infty$, $\operatorname{Re} \eta>-1 / p$, then

$$
\int_{0}^{\infty}\left|K_{\eta, \alpha}^{-} f(x)-f(x)\right|^{p} d x \rightarrow 0
$$

whenever $\alpha \rightarrow 0$ with the restriction $|\arg \alpha| \leqslant \theta<\pi / 2$ (Theorem VI in [17]).

In the first lemma, we obtain a more concrete representation of the operators $\left(I^{\alpha}\right)^{*}$. Let

$$
\Re=\left\{f \in L^{q}(0, \infty) \mid x^{k} f(x) \in L^{q}(0, \infty), \forall k \in Z^{+}\right\} .
$$

Clearly $\mathbb{N}$ is dense in $L^{q}(0, \infty), 1<q<\infty$.

LeMma 3.6. Let $\alpha>0$, and $\left(I^{\alpha}\right)^{*}$ be defined in $L^{q}(0, \infty), 1<q<\infty$, with domain (3.5.4). Then $\Re \subset D\left(\left(I^{\alpha}\right)^{*}\right)$, and for $f \in \Re$,

$$
\left(I^{\alpha}\right)^{*} f(x)=\frac{1}{\Gamma(\alpha)} \int_{x}^{\infty}(t-x)^{\alpha-1} f(t) d t
$$

the classical Weyl fractional integral. Moreover, $\left(I^{\alpha}\right)^{*} f \in \mathfrak{T}$.

Proof. Fix $f \in \Re$, and denote the integral in (3.6.1) by $J^{\alpha} f$. Since $t^{\alpha} f(t) \in L^{q}(0, \infty)$, by setting $\eta=0$ in (3.5.5) we see that $J^{\alpha} f(x)$ $=K_{0, \alpha}^{-}\left(t^{\alpha} f(t)\right)(x)$ a.e. in $(0, \infty)$. Then by (3.5.6), $J^{\alpha} f \in L^{q}(0, \infty)$. We show that $f \in D\left(\left(I^{\alpha}\right)^{*}\right)$, and $\left(I^{\alpha}\right)^{*} f=J^{\alpha} f$.

Let $g \in L^{p}(0, \infty), p=q /(q-1)$, and suppose $g \in D\left(I^{\alpha}\right)$. Then

$$
\int_{0}^{\infty} f(x) I^{\alpha} g(x) d x=\int_{0}^{\infty} g(x) J^{\alpha} f(x) d x ;
$$


the interchange of the order of integration is justified, for $f \in \Re$ implies $|f|$ $\in \Re$, so that $J^{\alpha}|f| \in L^{q}(0, \infty)$, also by (3.5.6). Thus $f \in D\left(\left(I^{\alpha}\right)^{*}\right)$, and as elements of $L^{q}(0, \infty),\left(I^{\alpha}\right)^{*} f=J^{\alpha} f$.

To verify the last statement, we again use (3.5.6). For if $k \in Z^{+}, \alpha$ $>0$ and $f \in \Re$, then

$$
x^{k}\left(I^{\alpha}\right)^{*} f(x)=x^{k} J^{\alpha} f(x)=K_{k, \alpha}^{-}\left(t^{k+\alpha} f(t)\right)(x),
$$

which belongs to $L^{q}(0, \infty)$ by (3.5.6). Thus $\left(I^{\alpha}\right)^{*} f \in \Re$.

LEMMA 3.7. $\subset \subset \mathbf{D}$.

Proof. Let $f \in \Re$. Then by Lemma 3.6, $f \in \bigcap_{\alpha>0} D\left(\left(I^{\alpha}\right)^{*}\right)$, and $\left(I^{\alpha}\right)^{*} f$ $\in \mathfrak{T}$ for each $\alpha>0$. Thus $f \in \bigcap_{\alpha, \beta>0} D\left(\left(I^{\alpha}\right)^{*}\left(I^{\beta}\right)^{*}\right)$; moreover, for $\alpha, \beta$ $>0,\left(I^{\alpha}\right)^{*}\left(I^{\beta}\right)^{*} f=J^{\alpha} J^{\beta} f=J^{\alpha+\beta} f=\left(I^{\alpha+\beta}\right)^{*} f$, where the second equality follows from Fubini's theorem, since $J^{\alpha} J^{\beta}|f|(x)=J^{\alpha+\beta}|f|(x)$ exists a.e. in $(0, \infty)$.

To verify the continuity requirements of the set $\mathbf{D}$, observe that

$$
\left\|t^{\alpha} f-f\right\|_{L^{p}(0, \infty)} \rightarrow 0 \text { as } \alpha \rightarrow 0^{+} .
$$

Then since $\left\|K_{0, \alpha}^{-}\right\| \leqslant M$, where $M$ is independent of $\alpha$ (by (3.5.7) and the uniform boundedness theorem),

$$
\begin{aligned}
\left\|\left(I^{\alpha}\right)^{*} f-f\right\| & =\left\|J^{\alpha} f-f\right\|=\left\|K_{0, \alpha}^{-}\left(t^{\alpha} f\right)-f\right\| \\
& \leqslant\left\|K_{0, \alpha}^{-}\left(t^{\alpha} f\right)-K_{0, \alpha}^{-}(f)\right\|+\left\|K_{0, \alpha}^{-}(f)-f\right\| \\
& \leqslant\left\|K_{0, \alpha}^{-}\right\|\left\|t^{\alpha} f-f\right\|+\left\|K_{0, \alpha}^{-}(f)-f\right\| \\
& \rightarrow 0 \quad \text { as } \alpha \rightarrow 0^{+}
\end{aligned}
$$

the term $\left\|K_{0, \alpha}^{-}(f)-f\right\|$ approaches zero as $\alpha \rightarrow 0^{+}$by (3.5.7).

Now for strong continuity at, say, $\beta>0$, we use the same argument as above, as well as the fact that $\left(I^{\beta}\right)^{*} f \in \Re$ if $f \in \Re$. Thus $\Re \subset \mathbf{D}$, as wanted.

For each $n \in Z^{+}$, let $\varphi_{n}(x)=e^{-n x}$. Then the set $\left\{\varphi_{n}\right\}_{n \in Z^{+}}$is fundamental in $L^{q}(0, \infty), 1<q<\infty$.

LEMMA 3.8. $\left\{\varphi_{n}\right\}_{n \in Z^{+}} \subset \Sigma$.

Proof. Since $\left\{\varphi_{n}\right\}_{n \in Z^{+}} \subset \Re$, we have by Lemma 3.7 that $\left\{\varphi_{n}\right\}_{n \in Z^{+}} \subset \mathbf{D}$. So it remains to show that, for each $n \in Z^{+}, \varphi_{n} \in \Sigma_{\omega}$ for suitable $\omega \in R$.

Fix $n \in Z^{+}$, and let $\alpha>0$. Then $\alpha=[\alpha]+\{\alpha\}$, where $[\alpha]$ is the greatest integer $\leqslant \alpha$, and $0 \leqslant\{\alpha\}<1$; hence 


$$
\begin{aligned}
\left\|\left(I^{\alpha}\right)^{*}\left(\varphi_{n}\right)\right\| & =\left\|J^{\alpha}\left(\varphi_{n}\right)\right\|=\left\|J^{\{\alpha\}} J^{[\alpha]}\left(\varphi_{n}\right)\right\| \\
& =\left\|J^{\{\alpha\}}\left(\varphi_{n} / n^{[\alpha]}\right)\right\|=\frac{1}{n^{[\alpha]}}\left\|J^{\{\alpha\}}\left(\varphi_{n}\right)\right\| \\
& \leqslant M / n^{[\alpha]}
\end{aligned}
$$

by the strong continuity of $J^{\{\alpha\}}\left(\varphi_{n}\right)$. Thus

$$
\left\|\left(I^{\alpha}\right)^{*}\left(\varphi_{n}\right)\right\| \leqslant M / n^{\alpha-1}=K e^{\omega \alpha},
$$

for suitable constants $K$ and $\omega \in R$, and so $\varphi_{n} \in \Sigma_{\omega}$.

Since $\left\{\varphi_{n}\right\}_{n \in Z^{+}}$is fundamental in $L^{q}(0, \infty)$, we now have that $\bar{\Sigma}=L^{q}(0, \infty)$, and applying Theorem 2.9(iv), we obtain

THEOREM 3.9. The infinitesimal generator of $\left\{\left(I^{\alpha}\right)^{*}\right\}_{\alpha>0}$ is densely defined.

As an operator in $L^{p}(0, \infty)$, the fractional derivative of order $\alpha, \alpha>0$, may be defined as the inverse of the operator $I^{\alpha}$. Another possibility is to define fractional derivatives in terms of the Weyl integral (3.4.1). (For these and other approaches, cf. Hardy, Landau and Littlewood [10], Kober [16], Love [19], and Tamarkin [22]).

Before giving another application of our theory, we return to the development of the preceding section. Let $C_{c}^{\infty}(0, \infty)$ denote the set of infinitely differentiable functions on $(0, \infty)$ with compact support. Then it is easy to see that for each $\alpha>0, C_{c}^{\infty}(0, \infty) \subset$ Range $\left(I^{\alpha}\right)$; thus the operators $I^{\alpha}$ have

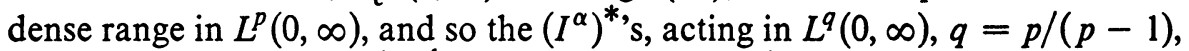
are 1-1. Therefore $\left[\left(I^{\alpha}\right)^{*}\right]^{-1}$, the inverse of $\left(I^{\alpha}\right)^{*}$, is a well-defined, closed operator in $L^{q}(0, \infty)$, with Domain $\left(\left[\left(I^{\alpha}\right)^{*}\right]^{-1}\right)=$ Range $\left(\left(I^{\alpha}\right)^{*}\right)$.

Now if $J_{\mathscr{N}}^{\alpha}$ denotes the Weyl integral (3.4.1) with domain the set $\Re$ (cf. (3.5.8)), then by Lemma 3.6 we have $J_{\mathscr{N}}^{\alpha} \subset\left(I^{\alpha}\right)^{*}$. Thus $\left(J_{\mathscr{N}}^{\alpha}\right)^{-1} \subset\left[\left(I^{\alpha}\right)^{*}\right]^{-1}$, so that on Range $\left(J_{\mathscr{N}}^{\alpha}\right)$, the operator $\left[\left(I^{\alpha}\right)^{*}\right]^{-1}$ is simply the fractional derivative of order $\alpha$, defined in terms of the Weyl fractional integral. For $\alpha \in Z^{+},\left(J_{\Re}^{\alpha}\right)^{-1} f$ is the $\alpha$ th derivative of $f$.

For convenience, set $D^{\alpha}=\left[\left(I^{\alpha}\right)^{*}\right]^{-1}$. In this section, we show that $\left\{D^{\alpha}\right\}_{\alpha>0}$ is a semigroup of closed linear operators in $L^{q}(0, \infty)$ with densely defined infinitesimal generator. The sets $\mathbf{D}$ and $\Sigma_{\omega}, \omega \in R$, will have their usual meanings and, in addition, we let $\delta$ denote the Schwartz space of all functions $f \in C^{\infty}(0, \infty)$ such that

$$
\sup _{x \in(0, \infty)}\left|x^{k} f^{(n)}(x)\right|<\infty
$$

for each pair of nonnegative integers $k, n$. Note that $\delta \subset \Re$.

LEMMA 3.10. $\delta \subset \mathbf{D}$. 
Proof. We first observe that, for each $\alpha>0, \delta \subset$ Range $\left(J_{\Re}^{\alpha}\right)$ (and certainly the latter is contained in Domain $\left(D^{\alpha}\right)$ ). For if $f \in \delta$, and $\alpha>0$, then we may write $f=J^{[\alpha]+1} g$, where $[\alpha]$ is the greatest integer $\leqslant \alpha$, and $g$ $=(-1)^{[\alpha]+1} f^{([\alpha]+1)}$, the $([\alpha]+1)$ th derivative of $f$. But $g \in \mathcal{S} \subset \mathfrak{N}$, so we have

$$
f=J^{[\alpha]+1} g=J^{\alpha} J^{[\alpha]+1-\alpha} g
$$

since $J^{[\alpha]+1-\alpha} g \in \Re$ by Lemma 3.6, $f \in$ Range $\left(J_{\Re(\alpha)}^{\alpha}\right.$.

In Lemma 3.7, we proved that if $f \in \mathscr{N}, J^{\alpha} J^{\beta} f=J^{\alpha+\beta}$. Thus $J_{\mathscr{N}}^{\alpha} J_{\mathscr{N}}^{\beta}$ $=J_{\Re}^{\alpha+\beta}$, as operators on $\Re$, so that we also have

$$
\delta \subset \text { Range }\left(J_{\mathscr{N}}^{\alpha} J_{\mathscr{N}}^{\beta}\right) \subset \text { Domain }\left(D^{\alpha} D^{\beta}\right) \text { for any } \alpha, \beta>0 .
$$

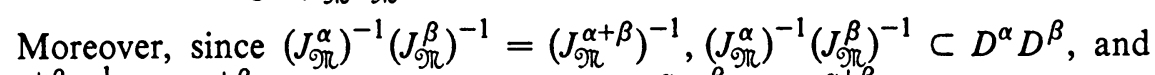
$\left(J_{\mathscr{\Omega}}^{\alpha+\beta}\right)^{-1} \subset D^{\alpha+\beta}$, we have for $f \in \delta$ that $D^{\alpha} D^{\beta} f=D^{\alpha+\beta} f$.

Next we check the continuity properties. If $f \in \delta$, then $f=J^{1} g\left(=J_{\Re}^{1} g\right)$, where $g \in \Re$. Thus for $0<\alpha<1$,

$$
\begin{aligned}
\left\|D^{\alpha} f-f\right\| & =\left\|D^{\alpha} J_{\Re}^{1} g-J_{\Re}^{1} g\right\|=\left\|D^{\alpha} J_{\Re}^{\alpha} J_{\Re}^{1-\alpha} g-J_{\Re}^{1} g\right\| \\
& =\left\|J_{\Re}^{1-\alpha} g-J_{\Re}^{1} g\right\| \rightarrow 0 \quad \text { as } \alpha \rightarrow 0^{+}
\end{aligned}
$$

(and as $\alpha \rightarrow 0^{-}$), by the strong continuity of $\left\{\left(I^{\alpha}\right)^{*}\right\}_{\alpha>0}$ on the set $\Re$ (cf. Lemma 3.7).

Similarly, for fixed $\beta>0$, we have $f=J^{\beta+1} h=J_{\Re}^{\beta+1} h$, where $h \in \Re$ (indeed, write $f=J_{\Re}^{[\beta]+2} g=J_{\mathscr{N}}^{\beta+1} J_{\mathscr{N}}^{[\beta]-\beta+1} g$ ). Then for $0<\alpha<1$,

$$
\begin{aligned}
\left\|D^{\alpha+\beta} f-D^{\beta} f\right\| & =\left\|D^{\alpha+\beta} J_{\Re}^{\beta+1} h-D^{\beta} J_{\Re}^{\beta+1} h\right\|=\left\|D^{\alpha+\beta} J_{\Re}^{\alpha+\beta} J_{\Re}^{1-\alpha} h-J_{\mathscr{N}}^{1} h\right\| \\
& =\left\|J_{\Re}^{1-\alpha} h-J_{\Re}^{1} h\right\| \rightarrow 0 \text { as } \alpha \rightarrow 0,
\end{aligned}
$$

again by Lemma 3.7. Thus $\delta \subset \mathbf{D}$, as claimed.

We again set $\varphi_{n}(x)=e^{-n x}$, for $n \in Z^{+}$. Then we have

LeMma 3.11. $\left\{\varphi_{n}\right\}_{n \in Z^{+}} \subset \Sigma$.

PRoof. Clearly $\left\{\varphi_{n}\right\}_{n \in Z^{+}} \subset \delta$, so by Lemma 3.10, $\left\{\varphi_{n}\right\}_{n \in Z^{+}} \subset$ D. Now if we fix $n \in Z^{+}$, and $\alpha>0$, then writing $\alpha=[\alpha]+\{\alpha\}$, we have (since $\varphi_{n} \in \mathcal{S} \subset$ Range $\left.\left(J_{\mathscr{N}}^{\alpha}\right)\right)$

$$
\begin{aligned}
& \left\|D^{\alpha}\left(\varphi_{n}\right)\right\|=\left\|\left(J_{\Re \Re}^{\alpha}\right)^{-1}\left(\varphi_{n}\right)\right\|=\left\|\left(J_{\Re R}^{\{\alpha\}}\right)^{-1}\left(J_{\Re R}^{[\alpha]}\right)^{-1}\left(\varphi_{n}\right)\right\|
\end{aligned}
$$

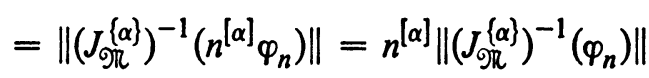

$$
\begin{aligned}
& \leqslant M n^{[\alpha]} \text {, }
\end{aligned}
$$


by the continuity of $\left(J_{\mathscr{R}}^{\alpha}\right)^{-1}\left(\varphi_{n}\right)$. Thus for suitable constants $K$ and $\omega \in R$, $\left\|D^{\alpha}\left(\varphi_{n}\right)\right\| \leqslant K e^{\alpha \omega}$, so $\varphi_{n} \in \Sigma_{\omega}$.

Again applying Theorem 2.11(iv), we have

THEOREM 3.12. The infinitesimal generator of $\left\{D^{\alpha}\right\}_{\alpha>0}$ is densely defined.

At the conclusion of $\$ 2$, we remarked that the infinitesimal generator of a semigroup of unbounded operators need not be densely defined. To confirm that this is indeed the case, we focus our attention on fractional derivatives in $L^{p}(0,1), 1<p<\infty$, in order to provide an example for which the infinitesimal generator is in fact the zero operator.

Let $\left\{I_{1}^{\alpha}\right\}_{\alpha>0}$ denote the Riemann-Liouville semigroup of bounded operators acting on $L^{p}(0,1)$. For $\alpha>0$, let

$$
D\left(I_{1}^{-\alpha}\right)=\left\{f \in L^{p}(0,1) \mid f=I_{1}^{\alpha} g \text { for some } g \in L^{p}(0,1)\right\},
$$

and define $I_{1}^{-\alpha} f=g$, if $f \in D\left(I_{1}^{-\alpha}\right)$, and $\alpha>0$. Since the operators $I_{1}^{\alpha}$ are $1-1, I_{1}^{-\alpha}$ is well defined; and for $\alpha \in Z^{+}, I_{1}^{-\alpha} f$ is simply the $\alpha$ th derivative of $f$.

As all operators considered will now act in $L^{\mu}(0,1)$, we shall write $I^{ \pm \alpha}$ for $I_{1}^{ \pm \alpha}$, and $\|\cdot\|$ for $\|\cdot\|_{L^{p}(0,1)}$.

As inverses of bounded operators on $L^{p}(0,1)$, the operators $I^{-\alpha}$ are closed, and satisfy the semigroup relation

$$
I^{-\alpha} I^{-\beta}=I^{-(\alpha+\beta)}, \quad \alpha, \beta>0 .
$$

Thus for $\alpha>\beta>0, D\left(I^{-\alpha}\right) \subset D\left(I^{-\beta}\right)$, and

$$
\bigcap_{\alpha>0} D\left(I^{-\alpha}\right)=\bigcap_{\alpha, \beta>0} D\left(I^{-\alpha} I^{-\beta}\right)=\bigcap_{n \in Z^{+}} D\left(I^{-n}\right) .
$$

Proposition 3.13. For each $\omega \in R, \Sigma_{\omega}=\{0\}$.

Proof. Fix $\omega \in R$, and let $f \in \Sigma_{\omega}$. Then in particular, $f \in \mathbf{D}$, so by (3.12.2), $f \in \cap_{n \in Z^{+}} D\left(I^{-n}\right)$. Thus $f \in C^{\infty}(0,1)$, and $f^{(k)}(0)=0$ for $k$ $\in Z^{+}$; also, $f(0)=0$. Moreover, there exists a constant $M$ such that $N_{\omega}(f)=\sup _{\alpha>0} e^{-\omega \alpha}\left\|I^{-\alpha} f\right\| \leqslant M$; thus for each integer $n \geqslant 1$,

$$
\left\|f^{(n)}\right\| \leqslant M e^{\omega n} \text {. }
$$

Now let $\varphi \in L^{q}(0,1)$, where $q=p /(p-1)$. Then integrating by parts, we have

$$
\begin{aligned}
\int_{0}^{1} \varphi(x) f(x) d x & =-\left.f(x) \int_{x}^{1} \varphi(t) d t\right|_{0} ^{1}+\int_{0}^{1} f^{\prime}(x) \int_{x}^{1} \varphi(t) d t d x \\
& =\int_{0}^{1} J^{1} \varphi(x) f^{\prime}(x) d x
\end{aligned}
$$


where $\left\{J^{\alpha}\right\}_{\alpha>0}$ denotes the semigroup $\left\{J_{1}^{\alpha}\right\}_{\alpha>0}$ of bounded operators on $L^{p}(0,1)$. Repeating (3.13.2) $n$ times, and using the fact that $f^{(k)}(0)=0$, we obtain

$$
\int_{0}^{1} \varphi(x) f(x) d x=\int_{0}^{1} J^{n} \varphi(x) f^{(n)}(x) d x
$$

for all $n \geqslant 0$. Thus by (3.13.1) and the usual bound on $J^{n}$,

$$
\begin{aligned}
\left|\int_{0}^{1} \varphi(x) f(x) d x\right| & \leqslant\left\|J^{n} \varphi\right\|_{L^{q}(0,1)}\left\|f^{(n)}\right\|_{L^{p}(0,1)} \\
& \leqslant \frac{M e^{\omega n}}{n !}\|\varphi\| \rightarrow 0 \text { as } n \rightarrow \infty .
\end{aligned}
$$

Thus $f \equiv 0$.

We now introduce a wide class of examples which illustrate our theory.

Fractional powers of closed operators have been defined in various ways (cf. Balakrishnan [2], Komatsu [18], Yosida [24, Chapter 9, §11], Berens, Butzer and Westphal [3]); we shall follow the development given by Balakrishnan [2].

Let $A$ be a closed linear operator acting in a Banach space $X$, and suppose the resolvent of $A$ satisfies

$$
\|\lambda R(\lambda ; A)\| \leqslant M, \text { for } \lambda>0 .
$$

Let $n \in Z^{+}$, and $\alpha \in C$ with $n-1<\operatorname{Re} \alpha<n$; define the linear operator $J^{\alpha}$ on $D\left(A^{n}\right)$, the domain of the operator $A^{n}$, by

$$
J^{\alpha} x=\frac{-\sin \alpha \pi}{\pi} \int_{0}^{\infty} \lambda^{\alpha-n} R(\lambda ; A) A^{n} x d \lambda
$$

where the principal value of $\lambda^{\alpha}$ is taken so that $\lambda^{\alpha}>0$ for $\alpha>0$. Then the following results are Lemmas 2.1, 2.2, and 2.4, respectively, in [2].

LEMMA 3.14. The operators $J^{\alpha}$ are closable.

Let $D\left(A^{\infty}\right)=\bigcap_{n \in Z^{+}} D\left(A^{n}\right)$.

LeMma 3.15. For $x \in D\left(A^{\infty}\right), J^{\alpha} x$ is an analytic function of $\alpha$ for $\operatorname{Re} \alpha>0$.

Lemma 3.16. For any $x \in D(A)$ such that $\lambda R(\lambda ; A) x \rightarrow 0$ as $\lambda \rightarrow 0, J^{\alpha} x$ $\rightarrow x$ as $\alpha \rightarrow 0^{+}$in a fixed sector about 0 .

For $\alpha \in Z^{+},(-A)^{\alpha}$ will denote the $\alpha$ th power of $(-A)$, defined in the usual way. For $\alpha \in C$ with $n-1<\operatorname{Re} \alpha<n$, where $n \in Z^{+}$, let $(-A)^{\alpha}$ denote the closure of the operator $J^{\alpha}$.

Then for $\alpha, \beta \in C$ with $\operatorname{Re} \alpha, \operatorname{Re} \beta>0$, and $x \in D\left(A^{\infty}\right)$, 


$$
(-A)^{\alpha}(-A)^{\beta} x=(-A)^{a+\beta} x
$$

(cf. [2, p. 423]).

If $A$ is the infinitesimal generator of a uniformly bounded semigroup of linear operators of class $\left(C_{0}\right)$ on $X$, then certainly $A$ is closed and satisfies (3.14.1). Moreover, by Lemma 3.15 and (3.16.1), $J^{\alpha} x$ is strongly continuous for $\alpha>0, x \in D\left(A^{\infty}\right)$; since the condition in Lemma 3.16 is satisfied for all $x \in A\left(D\left(A^{\infty}\right)\right)$, we see that $\left\{(-A)^{\alpha}\right\}_{\alpha>0}(\alpha$ now restricted to positive values) is a semigroup of closed linear operators in $X$, and $A\left(D\left(A^{\infty}\right)\right) \subset \mathbf{D}$, where $\mathbf{D}$ is the set defined in (2.1.1).

Even though $D\left(A^{\infty}\right)$ is dense in $X$ (cf. [12, Theorem 10.3.4]), we cannot conclude in general that such a semigroup has a densely defined infinitesimal generator. However, it is easy to see that the infinitesimal generator of $\left\{(-A)^{\alpha}\right\}_{\alpha}>0$ is dense in $X$ if the set of eigenvectors of $A$ (necessarily $\subset D\left(A^{\infty}\right)$ ) is fundamental, since any eigenvector $x$ belongs to $\Sigma_{\omega}$ for suitable $\omega \in R$. This condition agrees with the results on fractional derivatives in $L^{p}(0, \infty)$.

\section{REFERENCES}

1. W. G. Bade, Unbounded spectral operators, Pacific J. Math. 4 (1954), 373-392. MR 16, 143.

2. A. V. Balakrishnan, Fractional powers of closed operators and the semigroups generated by them, Pacific J. Math. 10 (1960), 419-437. MR 22 \#5899.

3. H. Berens, P. L. Butzer and U. Westphal, Representation of fractional powers of infinitesimal generators of semigroups, Bull. Amer. Math. Soc. 74 (1968), 191-196. MR 36 \#3163.

4. H. Berens and U. Westphal, A Cauchy problem for a generalized wave equation, Acta Sci. Math. (Szeged) 29 (1968), 93-106. MR 39 \#819.

5. A. Devinatz, A note on semi-groups of unbounded self-adjoint operators, Proc. Amer. Math. Soc. 5 (1954), 101-102. MR 15, 632.

6. N. Dunford and J. T. Schwartz, Linear operators. Parts I, II, III, Pure and Appl. Math., vol. 7, Interscience, New York, 1958, 1963, 1971. MR 22 \#8302; 32 \#6181.

7. W. Feller, Semi-groups of transformations in general weak topologies, Ann. of Math. (2) 57 (1953), 287-308. MR 14, 881.

8. , On the generation of unbounded semi-groups of bounded linear operators, Ann. of Math. (2) 58 (1953), 166 -174. MR 14, 1093.

9. M. J. Fisher, Imaginary powers of the indefinite integral, Amer. J. Math. 93 (1971), 317-328. MR 44 \# 7378.

10. G. H. Hardy, E. Landau and J. E. Littlewood, Some inequalities satisfied by the integrals or derivatives of real or analytic functions, Math. Z. 39 (1935), 677-695.

11. G. H. Hardy and J. E. Littlewood, Some properties of fractional integrals, Math. Z. 27 (1928), 565-606.

12. E. Hille and R. S. Phillips, Functional analysis and semi-groups, rev. ed., Amer. Math. Soc. Colloq. Publ., vol. 31, Amer. Math. Soc., Providence, R. I., 1957. MR 19, 664.

13. G. K. Kalisch, On fractional integrals of pure imaginary order in $L_{p}$, Proc. Amer. Math. Soc. 18 (1967), 136-139. MR 35 \#7145.

14. S. Kantorovitz, The semi-simplicity manifold of arbitrary operators, Trans. Amer. Math. Soc. 123 (1966), 241-252. MR 33 \# 572.

15. T. Kato, Perturbation theory for linear operators, Springer-Verlag, New York, 1966. MR 34 $\# 3324$. 
16. H. Kober, On fractional integrals and derivatives, Quart. J. Math. Oxford Ser. 11 (1940), 193-211. MR 2, 191.

17. - On a theorem of Schur and on fractional integrals of purely imaginary order, Trans. Amer. Math. Soc. 50 (1941), 160-174. MR 3, 39.

18. H. Komatsu, Fractional powers of operators, Pacific J. Math. 19 (1966), 285-346. 34 \# 1862.

19. E. R. Love, Two more hypergeometric integral equations, Proc. Cambridge Philos. Soc. 63 (1967), 1055-1076. MR 35 \#6874.

20. A. E. Nussbaum, Integral representation of semigroups of unbounded self-adjoint operators, Ann. of Math. (2) 69 (1959), 133-141. MR 21 \#305.

21. - Spectral representation of certain one-parametric families of symmetric operators in Hilbert space, Trans. Amer. Math. Soc. 152 (1970), 419-429. MR 42 \#3616.

22. J. D. Tamarkin, On integrable solutions of Abel's integral equation, Ann. of Math. (2) 31 (1930), 219-228.

23. D. V. Widder, The Laplace transform, Princeton Math. Ser., vol.6, Princeton Univ. Press, Princeton, N. J., 1941. MR 3, 232.

24. K. Yosida, Functional analysis, 4th ed., Die Grundlehren der math. Wissenschaften, Band 123, Springer-Verlag, New York, 1974. MR 50 \#2851.

Department of Mathematics, University of Illinois at Chicago Circle, Chicago, ILLINOIS 60680

Current address: Department of Mathematics, Tufts University, Medford, Massachusetts 02155 